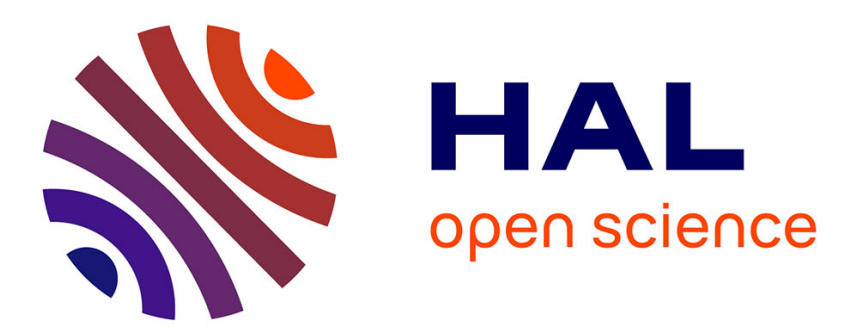

\title{
Bit Error Probability of Space Shift Keying MIMO over Multiple-Access Independent Fading Channels
}

\author{
Marco Di Renzo, Harald Haas
}

\section{To cite this version:}

Marco Di Renzo, Harald Haas. Bit Error Probability of Space Shift Keying MIMO over MultipleAccess Independent Fading Channels. IEEE Transactions on Vehicular Technology, 2011, 60 (8), pp.3694-3711. 10.1109/TVT.2011.2167636 . hal-00658684

\section{HAL Id: hal-00658684}

https://hal-centralesupelec.archives-ouvertes.fr/hal-00658684

Submitted on 20 Jan 2012

HAL is a multi-disciplinary open access archive for the deposit and dissemination of scientific research documents, whether they are published or not. The documents may come from teaching and research institutions in France or abroad, or from public or private research centers.
L'archive ouverte pluridisciplinaire HAL, est destinée au dépôt et à la diffusion de documents scientifiques de niveau recherche, publiés ou non, émanant des établissements d'enseignement et de recherche français ou étrangers, des laboratoires publics ou privés. 


\title{
Bit Error Probability of Space Shift Keying MIMO over Multiple-Access Independent Fading Channels
}

\author{
Marco Di Renzo, Member, IEEE and Harald Haas, Member, IEEE
}

\begin{abstract}
In this paper, we study the performance of Space Shift Keying (SSK) modulation for Multiple-Input-MultipleOutput (MIMO) wireless systems in the presence of multipleaccess interference. More specifically, a synchronous multi-user scenario is considered. The main technical contributions of this paper are as follows. Two receiver structures based on the Maximum-Likelihood (ML) criterion of optimality are developed and analytically studied, i.e., the single- and multi-user detectors. Accurate frameworks to compute the Average Bit Error Probability (ABEP) over independent and identically distributed (i.i.d.) Rayleigh fading channels are proposed. Furthermore, simple and easy-to-use lower- and upper-bounds for performance analysis and system design are introduced. The frameworks account for the near-far effect, which significantly affects the achievable performance in multiple-access environments. Also, we extend the analysis to Generalized SSK (GSSK) modulation, which foresees multiple-active antennas at the transmitter. With respect to SSK modulation, GSSK modulation achieves higher data rates at the cost of an increased complexity at the transmitter. The performance of SSK and GSSK modulations is compared to conventional Phase Shift Keying (PSK) and Quadrature Amplitude Modulation (QAM) schemes, and it is shown that SSK and GSSK modulations can outperform conventional schemes for various system setups and channel conditions. In particular, the performance gain of SSK and GSSK modulations increases for increasing values of the target bit rate and of the number of antennas at the receiver. Finally, we put forth the concept of Coordinated Multi-Point (or network MIMO) SSK (CoMPSSK) modulation, as a way of exploiting network cooperation and the spatial-constellation diagram to achieve high data rates. Analytical derivations and theoretical findings are substantiated through extensive Monte Carlo simulations for many setups.
\end{abstract}

Index Terms-Multiple-input-multiple-output (MIMO) wireless systems, space shift keying (SSK) modulation, spatial modulation (SM), multiple-access fading channels, performance analysis, bounds.

\section{INTRODUCTION}

$\mathbf{S}$ PACE SHIFT KEYING (SSK) is a recently proposed modulation scheme for Multiple-Input-Multiple-Output (MIMO) wireless systems [1], [2]. It encodes the information

Manuscript received May 19, 2011; revised August 3, 2011; accepted August 24, 2011. This paper was presented in part at the IEEE Global Communications Conference (GLOBECOM), Miami, FL, USA, December 2010. The review of this paper was coordinated by Prof. D. Matolak.

M. Di Renzo is with the Laboratoire des Signaux et Systèmes, Unité Mixte de Recherche 8506, Centre National de la Recherche ScientifiqueÉcole Supérieure d'Électricité-Université Paris-Sud XI, 91192 Gif-surYvette Cedex, France, (e-mail: marco.direnzo@lss.supelec.fr).

H. Haas is with The University of Edinburgh, College of Science and Engineering, School of Engineering, Institute for Digital Communications (IDCOM), King's Buildings, Mayfield Road, Edinburgh, EH9 3JL, United Kingdom (UK), (e-mail: h.haas@ed.ac.uk).

Color versions of one or more of the figures in this paper are available online at http://ieeexplore.ieee.org.

Digital Object Identifier XXX.XXX/TVT.XXX.XXX bits onto the spatial position (i.e., the index) of the antennas at the transmitter, and enables data decoding by exploiting the differences in the Channel Impulse Responses (CIRs) of the transmit-to-receive wireless links [3]. It is receiving an increasing attention due to its simple transmitter and receiver design, and, more important, because it represents the fundamental building block of Spatial Modulation (SM) [4][7]. SM is a low-complexity hybrid modulation scheme for MIMO systems, which maps the information bits onto two information carrying units: the signal-constellation diagram, which is determined by conventional modulation schemes (e.g., Phase Shift Keying (PSK) and Quadrature Amplitude Modulation (QAM)), and the spatial-constellation diagram, which is determined by SSK modulation [6]. By exploiting signal- and spatial-constellation diagrams, SM introduces a multiplexing gain with respect to single-antenna systems that increases logarithmically with the number of antennas at the transmitter. Furthermore, with respect to spatial-multiplexing MIMO systems, the multiplexing gain is obtained with no inter-channel interference. This enables very simple singlestream and Maximum-Likelihood (ML-) optimum decoding at the receiver [2], [6], [7]. Recent analytical and simulation results have highlighted that SM and SSK modulation can provide better performance with reduced decoding complexity than state-of-the-art single- and multi-antenna wireless systems [2], [5]-[15]. The reader can find a comprehensive and detailed overview of the contribution of recent papers on SM and SSK modulation in [3], [13], and [16].

By carefully looking at recent research works on SM and SSK modulation, it is possible to notice that all the studies available so far consider the point-to-point reference scenario. For example, in [2] and [7], the Average Bit Error Probability (ABEP) of SSK modulation and SM, respectively, is studied over independent and identically distributed (i.i.d.) Rayleigh fading channels; in [8], the framework in [2] is generalized to the so-called Generalized SSK (GSSK) modulation, which is an improved version of SSK modulation where more than one antenna can be active at any time instance; in [10], the study in [2] is extended by taking into account channel (Trellis) coding to reduce the error probability of detecting the active transmit-antenna; in [11], the performance of SM over Nakagami- $m$ fading channels is investigated; in [13], the ABEP of SSK modulation with and without transmit-diversity is analyzed over generically-correlated and distributed Rician fading channels; and, finally, in [3], [16], [17], the performance of SSK modulation is analyzed over correlated and nonidentically distributed Nakagami- $m$ fading with and without perfect Channel State Information (CSI). However, to the best of the authors knowledge, none of these papers address 
receiver design and performance analysis of SSK modulation in the presence of multiple-access interference. The present research work is motivated by the fact that, due to the simultaneous transmission of various users over the same physical wireless channel, the vast majority of wireless communication networks are interference limited [18]. Therefore, the potential merits of SSK modulation for its application to the next generation wireless communication systems highly depends on its robustness to multiple-access interference. The main aim of this paper is to understand advantages and disadvantages of SSK modulation in multiple-access environments (i.e., multiuser SSK modulation), and figure out if the claimed benefits of SSK modulation in a single-user environment are retained. Similar to [2], [3], and [16], we focus our attention only on SSK modulation as it enables a simple analytical derivation and insightful understanding of the role played by the spatialconstellation diagram, which is the main innovative enabler for performance improvement of SM/GSSK/SSK modulation [12]. In this context, we feel important to notice that the adoption of SM/GSSK/SSK modulation schemes inherently requires that each user is equipped with multiple antennas at the transmitter and at the receiver. Also, since in some cases, e.g., in SSK modulation, only a single antenna is active and the information is conveyed only by the spatial-constellation diagram, the number of antennas in each device might be quite large to achieve good data rates. However, this large number of antennas seems not to be a critical bottleneck for the development of the next generation multiple-access cellular systems, as current research is moving towards the utilization of the millimeter-wave frequency spectrum [19]. In fact, in this band compact horn antenna-arrays with 48 elements and compact patch antenna-arrays with more than 4 elements at the Base Station (BS) and at the mobile terminal, respectively, are currently being developed to support multigigabit transmission rates [20].

In detail, the main contributions and technical novelties of this paper can be summarized as follows: i) for the first time, the performance of modulation schemes exploiting the "space modulation" concept in a multi-user interference environment is investigated and compared to traditional modulation schemes; ii) two ML-optimum receiver structures are investigated, i.e., the single-user detector that is interferenceunaware and the joint multi-user detector that is interferenceaware; iii) for analytical tractability, we limit our performance study to the synchronous case, which is often considered as the initial reference scenario for performance analysis in the presence of interference [21]; iv) accurate frameworks for SSK and GSSK modulations are developed to compute the ABEP over i.i.d. frequency-flat Rayleigh fading channels. The frameworks can take into account the so-called near-far effect that significantly affects the performance of wireless systems in multiple-access environments [21]; v) simple bounds are derived to compare various modulation schemes (e.g., SSK, GSSK, PSK, QAM) and to understand advantages and disadvantages of each of them for various MIMO configurations; vi) the proposed frameworks and bounds are useful for an arbitrary number of antennas at the transmitter and at the receiver, as well as for any modulation order and bit-to- antenna-index mapping; and vii) we put forth the concept of Coordinated Multi-Point (or network MIMO) SSK (CoMPSSK) modulation, as a way of exploiting network cooperation and the spatial-constellation diagram to achieve high bit rates.

Our comprehensive analytical study highlights the following general results: i) it is shown that SSK and GSSK modulations can outperform conventional modulation schemes in the presence of multiple-access interference; ii) if a single-user detector is used, SSK modulation provides better performance than PSK modulation for bit rates greater than 2 bits $/ \mathrm{s} / \mathrm{Hz}$ per user, while it outperforms QAM for bit rates greater than $2 \mathrm{bits} / \mathrm{s} / \mathrm{Hz}$ per user if the receiver is equipped with at least two antennas; iii) GSSK modulation is always worse than SSK modulation, but it achieves higher bit rates for the same number of antennas at the transmitter; iv) the performance gain of SSK and GSSK modulations increases for increasing values of the requested bit rate per user; v) the robustness of SSK and GSSK modulations to multi-user interference increases by adding more antennas at the receiver; vi) when a multiuser detector is used, SSK and GSSK modulations seem to be more robust to multi-user interference than conventional modulation schemes. For example, for bit rates greater than 2 bits/s/Hz per user, SSK modulation always outperforms QAM regardless of the number of antennas at the receiver; and vii) it is shown that CoMP-SSK modulation can provide very high bit rates at the cost of network cooperation, which can be realized through a backhaul link. Also, it is shown that CSI at the transmitter is not needed to implement this scheme.

The reminder of this paper is organized as follows. In Section II, the system model is introduced. In Section III, the ABEP of SSK modulation with ML-optimum single-user detection is investigated. In Section IV, the performance of SSK modulation is compared to QAM and PSK modulation when a ML-optimum single-user detector is used. In Section V, the framework in Section III is extended to GSSK modulation, and SSK and GSSK modulations are compared. In Section VI, all the frameworks proposed in the previous sections are generalized to ML-optimum multi-user detection, and asymptotic analysis is used to reveal advantages and disadvantages of SSK and GSSK modulations. In Section VII, the concept of CoMP-SSK modulation is introduced. In Section VIII, numerical results are shown to substantiate our analytical derivations and findings. Finally, Section IX concludes this paper.

\section{System Model}

We consider a very general multi-user MIMO setup with $N_{u}$ active users (i.e., transmitters) and $N_{d}$ possible receivers. Each transmitter/receiver is equipped with an antenna-array of $N_{t} / N_{r}$ antennas. This setup can accommodate various multiple-access situations, such as: i) the scenario in which a single receiver must decode the information of more than one transmitter, and ii) the so-called interference channel (e.g., the $X$ channel) [18], where each receiver must decode the information from a single transmitter and can disregard the information transmitted by the other users. Without loss of generality, among the possible transmitter/receiver pairs, 


$$
z^{(r)}(t)=\underbrace{\sqrt{E_{\xi}} h_{\xi}^{\left(x_{\xi}, r\right)} w(t)}_{\text {probe link }}+\underbrace{\sum_{\substack{u=1 \\ u \neq \xi}}^{N_{u}}\left[\sqrt{E_{u}} h_{u}^{\left(x_{u}, r\right)} w(t)\right]}_{\text {interference }}+\underbrace{n^{(r)}(t)}_{\text {AWGN }}
$$

we focus our attention on a particular link that is usually known as probe link or intended link (see, e.g., [22, Fig. $1])$. More specifically, we are interested in studying the error probability of the data sent from a generic user $\xi$, with $\xi=$ $1,2, \ldots, N_{u}$, to a generic receiver when the other $N_{u}-1$ users are simultaneously transmitting on the same physical channel. So, there is no loss of generality in considering $N_{d}=1$.

In our system model, the $N_{u}$ users exploit the differences (i.e., known as spatial signatures or channel fingerprints [3]) in the wireless channel of any transmit-to-receive link with a twofold objective: data modulation and multipleaccess. Accordingly, the multi-user SSK modulation scheme analyzed in this paper can be seen as a generalization of the so-called Space-Division Multiple-Access (SDMA) [23] [25] and Channel-Division Multiple-Access (ChDMA) [26] schemes, which exploit user-specific CIRs only for differentiating simultaneously transmitting users, i.e., for multipleaccess only. The fundamental difference between multi-user SSK modulation and multi-user SDMA/ChDMA is that the former scheme uses the differences in the CIRs for data modulation in addition to multiple-access, while the latter schemes rely on conventional (e.g., PSK and QAM) modulation for transmitting the data of the users. In multi-user SSK modulation, the stochastic differences in the CIRs are exploited in a twofold way: i) at the microscopic level, i.e., differences among co-located transmit-antennas of the same user, for data modulation, and ii) at the macroscopic level, i.e., differences among spatially distributed antennaarrays associated to different users, for multiple-access. To the best of the authors knowledge, the performance analysis of this multiple-access scheme has never been considered in literature.

\section{A. Assumptions and Notation}

Throughout this paper, the following assumptions and notation are used. i) A synchronous multi-access channel with perfect time-synchronization at the receiver is considered [21]. Accordingly, for ease of notation, time delays can be neglected during the analytical derivation. ii) The receiver is assumed to have perfect CSI. More specifically, if a single-user detector is used, the receiver needs only the CSI of the probe link. While, if a joint multi-user detector is used, the receiver needs the CSI of all the active users. iii) In all wireless links, frequency-flat independent Rayleigh fading is assumed. In particular, identically distributed fading is considered for wireless links related to co-located antennas, while nonidentically distributed fading among the users is considered. This allows us to include the near-far effect in the analytical derivation. In formulas, we denote by $h_{u}^{(t, r)}$ the complex CIR from the $t$-th $\left(t=1,2, \ldots, N_{t}\right)$ transmit-antenna of the $u$-th $\left(u=1,2, \ldots, N_{u}\right)$ user to the $r$-th $\left(r=1,2, \ldots, N_{r}\right)$ receiveantenna of the destination. Moreover, we use the notation $h_{u}^{(t, r)}=\operatorname{Re}\left\{h_{u}^{(t, r)}\right\}+j \operatorname{Im}\left\{h_{u}^{(t, r)}\right\}$, where $\operatorname{Re}\{\cdot\}$ and $\operatorname{Im}\{\cdot\}$ are real and imaginary operators, and $j=\sqrt{-1}$ is the imaginary unit. $\operatorname{Re}\left\{h_{u}^{(t, r)}\right\}$ and $\operatorname{Im}\left\{h_{u}^{(t, r)}\right\}$ are independent real-valued Random Variables (RVs). iv) $X \sim \mathcal{N}\left(\mu, \sigma^{2}\right)$ denotes a Gaussian RV with mean $\mu$ and standard deviation $\sigma$. v) Owing to the assumption of Rayleigh fading, we have $\operatorname{Re}\left\{h_{u}^{(t, r)}\right\} \sim \mathcal{N}\left(0, \sigma_{u}^{2}\right)$ and $\operatorname{Im}\left\{h_{u}^{(t, r)}\right\} \sim \mathcal{N}\left(0, \sigma_{u}^{2}\right)$. vi $)$ $\overline{(\cdot)}$, i.e., overbar, denotes complex-conjugate. vii) $\mathrm{E}\{\cdot\}$ denotes the expectation operator. viii) $\operatorname{Pr}\{\cdot\}$ denotes probability. ix) $Q(x)=(1 / \sqrt{2 \pi}) \int_{x}^{+\infty} \exp \left(-t^{2} / 2\right) d t$ is the Q-function and $Q^{-1}(\cdot)$ is used to denoted the inverse Q-function. x) $T_{s}$ denotes the duration of the time-slot where each information symbol is transmitted. xi) The noise $n^{(r)}$ at the input of the $r$-th receive-antenna $\left(r=1,2, \ldots, N_{r}\right)$ is assumed to be an Additive White Gaussian Noise (AWGN) process, with both real and imaginary parts having a power spectral density equal to $N_{0}$. Across the receive-antennas, the noise is statistically independent. xii) $M$ denotes the modulation order of QAM and PSK modulation. The $M$ symbols of the signal-constellation diagram of user $u$ are denoted by the complex numbers $s_{u}^{(m)}$ for $m=1,2, \ldots, M$ and $u=1,2, \ldots, N_{u}$. For PSK modulation, we have $\left|s_{u}^{(m)}\right|^{2}=1$. xiii) If GSSK modulation is used, $N_{\text {ta }}$ denotes the number of simultaneously-active antennas at the transmitter, with $1 \leq N_{\mathrm{ta}} \leq N_{t}$. The effective size of the spatial-constellation diagram is denoted by $N=2^{\left\lfloor\log _{2}\left(\begin{array}{c}N_{t} \\ N_{\mathrm{ta}}\end{array}\right)\right\rfloor}$ [8], where $($.$) is the binomial coefficient and \lfloor\cdot\rfloor$ is the floor function. xiv) In SSK and GSSK modulations, $E_{u}$ and $E_{u} / N_{\text {ta }}$ denote the average energy transmitted by each antenna of user $u\left(u=1,2, \ldots, N_{u}\right)$ that emits a non-zero signal, respectively. In particular, in GSSK modulation a uniform energy-allocation scheme is considered among the active transmit-antennas. In QAM and PSK modulation, $E_{u}$ is the average transmitted energy per information symbol of user $u$. xv) $w(\cdot)$ is the real-valued unit-energy transmitted pulse shape in each timeslot $T_{s}$. xvi) $\Gamma(x)=\int_{0}^{+\infty} t^{z-1} \exp (-t) d t$ is the Gamma function. xvii) $\delta_{x, y}$ is the Kronecker delta function, which is defined as $\delta_{x, y}=1$ if $x=y$ and $\delta_{x, y}=0$ elsewhere. xviii) $\stackrel{(a)}{=}$, $\stackrel{(a)}{\leq}$, and $\stackrel{(a)}{\approx}$ denote conventional equality $(=)$, inequality $(\leq)$, and approximation $(\approx)$ operators, respectively, which have been labeled with $(a)$ as a short-hand to better identify them in the text, and provide comments on the analytical procedure that is used for their computation.

\section{SSK Modulation With Single-User Detection}

In SSK modulation, each user encodes blocks of $\log _{2}\left(N_{t}\right)$ data bits into the index of a single transmit-antenna, which is 


$$
\hat{x}_{\xi}=\underset{y_{\xi}=1,2, \ldots, N_{t}}{\arg \min }\left\{D_{\xi}^{\left(y_{\xi}\right)}\right\}=\underset{y_{\xi}=1,2, \ldots, N_{t}}{\arg \min }\left\{\sum_{r=1}^{N_{r}} \int_{T_{s}}\left|z^{(r)}(t)-\sqrt{E_{\xi}} h_{\xi}^{\left(y_{\xi}, r\right)} w(t)\right|^{2} d t\right\}
$$

$$
\begin{gathered}
\operatorname{ABEP}_{\xi} \stackrel{(a)}{=} \mathrm{E}_{\mathbf{h}}\left\{\frac{1}{N_{t}} \sum_{x_{\xi}=1}^{N_{t}}\left[\frac{1}{\log _{2}\left(N_{t}\right)} \sum_{y_{\xi}=1}^{N_{t}} N_{H}\left(x_{\xi}, y_{\xi}\right) \operatorname{Pr}\left\{\hat{x}_{\xi}=y_{\xi} \mid x_{\xi}\right\}\right]\right\} \\
\stackrel{(b)}{\leq} \frac{1}{N_{t}} \sum_{x_{\xi}=1}^{N_{t}}[\frac{1}{\log _{2}\left(N_{t}\right)} \sum_{y_{\xi}=1}^{N_{t}} N_{H}\left(x_{\xi}, y_{\xi}\right) \underbrace{\operatorname{E}_{\mathbf{h}}\left\{\operatorname{Pr}\left\{x_{\xi} \rightarrow y_{\xi}\right\}\right\}}_{\operatorname{APEP}\left(x_{\xi} \rightarrow y_{\xi}\right)}]
\end{gathered}
$$

$$
\operatorname{PEP}\left(x_{\xi} \rightarrow y_{\xi}\right) \stackrel{(a)}{=} \operatorname{Pr}\left\{D_{\xi}^{\left(x_{\xi}\right)}>D_{\xi}^{\left(y_{\xi}\right)}\right\} \stackrel{(b)}{=} \operatorname{Pr}\left\{2 \operatorname{Re}\left\{\sum_{r=1}^{N_{r}}\left[\sqrt{E_{\xi}}\left(\bar{h}_{\xi}^{\left(y_{\xi}, r\right)}-\bar{h}_{\xi}^{\left(x_{\xi}, r\right)}\right) \eta^{(r)}\right]\right\}>E_{\xi} \sum_{r=1}^{N_{r}}\left|h_{\xi}^{\left(y_{\xi}, r\right)}-h_{\xi}^{\left(x_{\xi}, r\right)}\right|^{2}\right\}
$$

$$
\Omega \sim \mathcal{N}\left(2 \sqrt{E_{\xi}} \operatorname{Re}\left\{\sum_{r=1}^{N_{r}}\left[\left(\bar{h}_{\xi}^{\left(y_{\xi}, r\right)}-\bar{h}_{\xi}^{\left(x_{\xi}, r\right)}\right) \sum_{u \neq \xi=1}^{N_{u}}\left(\sqrt{E_{u}} h_{u}^{\left(x_{u}, r\right)}\right)\right]\right\}, 4 N_{0} E_{\xi} \sum_{r=1}^{N_{r}}\left|h_{\xi}^{\left(y_{\xi}, r\right)}-h_{\xi}^{\left(x_{\xi}, r\right)}\right|^{2}\right)
$$

switched on for data transmission while all the other antennas are kept silent. Let $\xi$, for $\xi=1,2, \ldots, N_{u}$, be the user of interest, i.e., the probe link, while let all the other $N_{u}-1$ users be interfering users. Also, let $x_{u}$, for $x_{u}=1,2, \ldots, N_{t}$, be the antenna-index of the generic user $u$ that is actually switched on for transmission. Accordingly, the signal received after propagation through the wireless fading channel and impinging upon the $r$-th $\left(r=1,2, \ldots, N_{r}\right)$ receive-antenna is given in (1) on top of the previous page.

As mentioned in Section II, (1) confirms that in multiuser SSK modulation only the differences in the CIRs are exploited for modulation and multiple-access. All the users share the same time-slot, frequency-band, or spreading code [26]. By assuming single-user detection, the interference is not exploited for optimal detection, and the MLoptimum estimate, $\hat{x}_{\xi}$, of $x_{\xi}$ is shown in (2) on top of this page [27], where $y_{\xi}$ denotes the trial instance of $x_{\xi}$ used in the $N_{t}$-hypothesis testing problem, and $D_{\xi}^{\left(y_{\xi}\right)}=$ $\sum_{r=1}^{N_{r}} \int_{T_{s}}\left|z^{(r)}(t)-\sqrt{E_{\xi}} h_{\xi}^{\left(y_{\xi}, r\right)} w(t)\right|^{2} d t$.

The ABEP of the detector in (2) can be computed in closed-form as shown in (3) on top of this page, where $\stackrel{(a)}{=}$ comes from [28, Eq. (4) and Eq. (5)], and $\stackrel{(b)}{=}$ is the wellknown asymptotically-tight union-bound [29, Eq. (12.44)], which has recently been used in [3, Eq. (35)] for pointto-point SSK modulation. Furthermore, $N_{H}\left(x_{\xi}, y_{\xi}\right)$ is the Hamming distance between the bit-to-antenna-index mappings of $x_{\xi}$ and $y_{\xi} ; \mathrm{E}_{\mathbf{h}}\{\cdot\}$ is the expectation computed over all the fading channels (probe link and interference); and $\operatorname{APEP}\left(x_{\xi} \rightarrow y_{\xi}\right)=\mathrm{E}_{\mathbf{h}}\left\{\operatorname{PEP}\left(x_{\xi} \rightarrow y_{\xi}\right)\right\}$ is the Average Pairwise Error Probability (APEP), i.e., the probability of estimating $y_{\xi}$ when, instead, $x_{\xi}$ is transmitted, under the assumption that $x_{\xi}$ and $y_{\xi}$ are the only two antenna-indexes possibly being transmitted. Accordingly, $\operatorname{PEP}\left(x_{\xi} \rightarrow y_{\xi}\right)=$ $\operatorname{Pr}\left\{x_{\xi} \neq y_{\xi}\right\}$ is conditioned on both antenna-indexes $x_{\xi}$ and $y_{\xi}$. Unlike [3], the main contribution of this paper consists in taking into account multiple-access interference when computing the APEPs.

The PEPs in (3) can explicitly be written as shown in (4) on top of this page, where $\stackrel{(a)}{=}$ comes directly from $(2)$, and $\stackrel{(b)}{=}$ can be obtained after lengthly analytical manipulations. Furthermore, we have defined $\eta^{(r)}=\sum_{u \neq \xi=1}^{N_{u}}\left(\sqrt{E_{u}} h_{u}^{\left(x_{u}, r\right)}\right)+n_{w}^{(r)}$ and $n_{w}^{(r)}=\int_{T_{s}} n^{(r)}(t) \bar{w}(t) d t$. By direct inspection, it is easy to show that $n_{w}^{(r)}$ is a complex-valued Gaussian RV with distribution $\operatorname{Re}\left\{n_{w}^{(r)}\right\} \sim \mathcal{N}\left(0, N_{0}\right)$ and $\operatorname{Im}\left\{n_{w}^{(r)}\right\} \sim$ $\mathcal{N}\left(0, N_{0}\right)$.

By conditioning upon all the fading channels (probe link and interference), it can be proved that $\Omega=$ $2 \operatorname{Re}\left\{\sum_{r=1}^{N_{r}}\left[\sqrt{E_{\xi}}\left(\bar{h}_{\xi}^{\left(y_{\xi}, r\right)}-\bar{h}_{\xi}^{\left(x_{\xi}, r\right)}\right) \eta^{(r)}\right]\right\}$ in (4) is a realvalued Gaussian RV with distribution shown in (5) on top of this page. Thus, from the definition of Q-function in Section II-A, the PEP in (4) has closed-form expression given in (6) on top of the next page. It is worth mentioning that the PEP in (6) is very general and can be used for channel models different from Rayleigh fading studied in this paper. Furthermore, (6) is obtained without making any assumptions about the statistical distribution of the interference. This enables us to use, with minor changes, this formula for modulation schemes different from SSK, such as PSK/QAM and GSSK, which are studied in Section IV and Section V, respectively. Thus, the assumption of Rayleigh fading is here made only for analytical tractability, and to get simple and insightful closed-form expressions and bounds, which: i) enable a fairly simple comparison among different state-of-the-art modulation schemes; ii) provide guidelines for system design and optimization; and iii) shed 


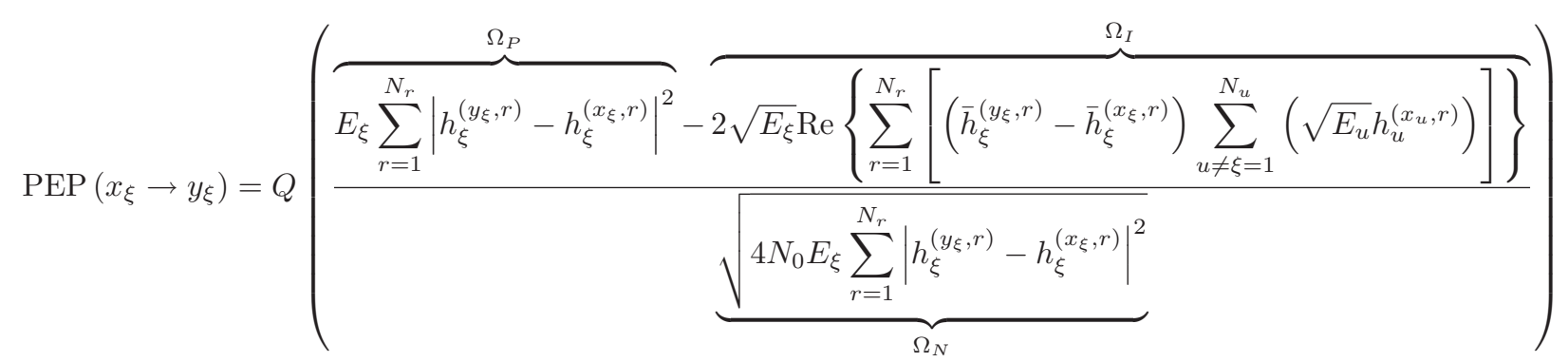

$$
\begin{aligned}
\operatorname{APEP}\left(x_{\xi} \rightarrow y_{\xi}\right) & =\mathrm{E}_{\mathbf{h}_{\xi}}\left\{Q\left(\frac{\Omega_{P}}{\sqrt{\Omega_{N}^{2}+\sigma_{I}^{2}}}\right)\right\}=\mathrm{E}_{\mathbf{h}_{\xi}}\left\{Q\left(\sqrt{\frac{\left(E_{\xi} / N_{0}\right) \gamma_{\xi}}{4+4 \sum_{u \neq \xi=1}^{N_{u}}\left[\left(E_{u} \sigma_{u}^{2}\right) / N_{0}\right]}}\right)\right\} \\
& \stackrel{(a)}{=}\left[\frac{1}{2}\left(1-\sqrt{\frac{\mathrm{SINR}}{2+\mathrm{SINR}}}\right)\right]^{N_{r}} \sum_{r=1}^{N_{r}}\left\{\left(\begin{array}{c}
N_{r}-1+r \\
r
\end{array}\right)\left[\frac{1}{2}\left(1+\sqrt{\frac{\mathrm{SINR}}{2+\mathrm{SINR}}}\right)\right]^{r}\right\}
\end{aligned}
$$

lights on the robustness of SSK modulation to multiple-access interference.

To compute the APEP in Rayleigh fading, i.e., to remove the conditioning over channel statistics, we use a two-step procedure: i) first, we condition the PEP in (6) upon the channel gains of the probe link and remove the conditioning over the channel gains of the interference; and ii) then, we remove the conditioning over the channel gains of the probe link. By conditioning upon the probe link, $\Omega_{I}$ in (6) is a conditional Gaussian RV with distribution $\Omega_{I} \sim$ $\mathcal{N}\left(0, \sigma_{I}^{2}=4 E_{\xi}\left[\sum_{u \neq \xi=1}^{N_{u}}\left(E_{u} \sigma_{u}^{2}\right)\right]\left[\sum_{r=1}^{N_{r}}\left|h_{\xi}^{\left(y_{\xi}, r\right)}-h_{\xi}^{\left(x_{\xi}, r\right)}\right|^{2}\right]\right)$, while $\Omega_{P}$ and $\Omega_{N}$ are conditional constant terms. Accordingly, from (6) we have:

$$
\begin{aligned}
\mathrm{E}_{\mathbf{h}_{\backslash \xi}}\left\{\operatorname{PEP}\left(x_{\xi} \rightarrow y_{\xi}\right)\right\} & \stackrel{(a)}{=} \mathrm{E}_{\mathbf{h}_{\backslash \xi}}\left\{Q\left(\frac{\Omega_{P}-\Omega_{I}}{\Omega_{N}}\right)\right\} \\
& \stackrel{(b)}{=} \mathrm{E}_{\mathbf{h}_{\backslash \xi}}\left\{Q\left(\frac{\Omega_{P}}{\Omega_{N}}-\frac{\sigma_{I}}{\Omega_{N}} \tilde{\Omega}_{I}\right)\right\} \\
& \stackrel{(c)}{=} Q\left(\frac{\Omega_{P}}{\sqrt{\Omega_{N}^{2}+\sigma_{I}^{2}}}\right)
\end{aligned}
$$

where $\mathrm{E}_{\mathbf{h}_{\backslash \xi}}\{\cdot\}$ denotes the expectation over all the fading gains except those of the probe link; $\stackrel{(a)}{=}$ comes from $(6) ; \stackrel{(b)}{=}$ is obtained by introducing the RV $\tilde{\Omega}_{I}$, which is defined as $\tilde{\Omega}_{I}=\Omega_{I} / \sigma_{I} \sim \mathcal{N}(0,1)$; and $\stackrel{(c)}{=}$ is a notable integral that involves the Q-function and Gaussian RVs, and is tabulated in [21, Eq. (3.66)].

The last step consists in removing the conditioning over the channel gains of the probe link. From (7), we obtain (8) on top of this page, where we have defined: i) $\gamma_{\xi}=$ $\sum_{r=1}^{N_{r}}\left|h_{\xi}^{\left(y_{\xi}, r\right)}-h_{\xi}^{\left(x_{\xi}, r\right)}\right|^{2}$; ii) $\mathrm{SINR}=\mathrm{SNR}_{\xi} /\left(1+\mathrm{INR}_{\backslash \xi}\right)$ is the Signal-to-(Interference+Noise)-Ratio (SINR); iii) $\mathrm{SNR}_{\xi}=\left(E_{\xi} \sigma_{\xi}^{2}\right) / N_{0}$ is the Signal-to-Noise-Ratio (SNR) of the probe link; and iv) $\operatorname{INR}_{\backslash \xi}=\sum_{u \neq \xi=1}^{N_{u}}\left[\left(E_{u} \sigma_{u}^{2}\right) / N_{0}\right]$ is the aggregate Interference-to-Noise-Ratio (INR) of all the interferers. The identity in $\stackrel{(a)}{=}$ is obtained as follows: i) $\gamma_{\xi}$ is the summation of the square absolute value of Gaussian RVs and, thus, is a Chi-Square RV [30, Eq. (2-1-136), Eq. (2-1137)]; and ii) the Q-function is averaged over the resulting Chi-Square RV [30, Eq. (14-4-14), Eq. (14-4-15)].

Finally, the ABEP can be computed by substituting (8) in (3). By carefully looking at (8), we notice that the APEP is independent of $x_{\xi}$ and $y_{\xi}$, i.e., the actual and trial antennaindexes, but it only depends on SNR and INR of probe link and interference, respectively. Thus, by defining in (8) $\operatorname{APEP}\left(x_{\xi} \rightarrow y_{\xi}\right)=\operatorname{APEP}_{\xi}$ for all $x_{\xi}=1,2, \ldots, N_{t}$ and $y_{\xi}=1,2, \ldots, N_{t}$, the ABEP in (3) simplifies as follows:

$$
\begin{aligned}
\operatorname{ABEP}_{\xi} & \leq \frac{\operatorname{APEP}_{\xi}}{N_{t} \log _{2}\left(N_{t}\right)} \sum_{x_{\xi}=1}^{N_{t}} \sum_{y_{\xi}=1}^{N_{t}} N_{H}\left(x_{\xi}, y_{\xi}\right) \\
& \stackrel{(a)}{=} \frac{N_{t}}{2} \operatorname{APEP}_{\xi}
\end{aligned}
$$

where $\stackrel{(a)}{=} \quad$ comes from the identity $\sum_{x_{\xi}=1}^{N_{t}} \sum_{y_{\xi}=1}^{N_{t}} N_{H}\left(x_{\xi}, y_{\xi}\right)=\left(N_{t}^{2} / 2\right) \log _{2}\left(N_{t}\right)$, which can be derived via direct inspection for all possible bit-toantenna-index mappings.

In conclusion, (8) and (9) provide a very simple analytical framework of the ABEP of multi-user SSK modulation over Rayleigh fading channels. Very interestingly, from (9) we observe that the ABEP is independent of the bit-toantenna-index mapping. This stems from the assumption of i.i.d. Rayleigh fading for the wireless links of co-located transmit-antennas. Also, we note that (8) and (9) reduce to known results if there is no multiple-access interference, i.e., $\mathrm{INR}_{\backslash \xi}=0$ (see, e.g., [2] and [3]).

\section{A. Asymptotic Analysis}

In this section, we study some asymptotic case studies to highlight some general behaviors when using the spatialconstellation diagram for modulation.

1) $\mathrm{SNR}_{\xi} \gg 1$ and $\mathrm{INR}_{\backslash \xi} \ll 1$ (noise-limited scenario): This corresponds to a scenario in which multi-access interference can be neglected and large-SNR analysis for the probe 


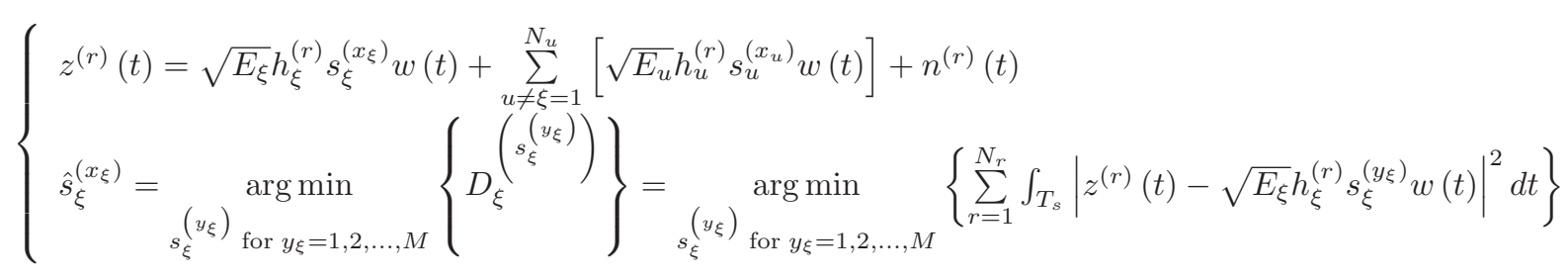

link can be considered. In this case, from [30, Sec. 14-5-3] the ABEP turns out to be asymptotically equal to $\mathrm{ABEP}_{\xi} \rightarrow$ $2^{-\left(N_{r}+1\right)}\left(\begin{array}{c}2 N_{r}-1 \\ N_{r}\end{array}\right) N_{t} \mathrm{SNR}_{\xi}^{-N_{r}}$. This formula shows that, if the multiple-access interference is negligible, the ABEP goes to zero for increasing SNR and that the diversity order is equal to $N_{r}$. These findings agree with [2] and [3]. This formula highlights a trend that was not shown either in [2] or [3]: the ABEP linearly increases with the number of transmit-antennas $N_{t}$. As the bit rate increases with $\log _{2}\left(N_{t}\right)$, there is a tradeoff to consider.

2) $\mathrm{INR}_{\backslash \xi} \gg 1$ and $\mathrm{SIR}=\mathrm{SNR}_{\xi} / \mathrm{INR}_{\backslash \xi} \gg 1$ (interference-limited scenario): This corresponds to a scenario in which the AWGN is negligible, and the Signal-to-Interference-Ratio (SIR) is high. In this case, the ABEP is asymptotically equal to $\mathrm{ABEP}_{\xi} \rightarrow 2^{-\left(N_{r}+1\right)}\left(\begin{array}{c}2 N_{r}-1 \\ N_{r}\end{array}\right) N_{t} \mathrm{SIR}^{-N_{r}} \quad$ with $\mathrm{SIR}=E_{\xi} \sigma_{\xi}^{2} / \sum_{u \neq \xi=1}^{N_{u}}\left(E_{u} \sigma_{u}^{2}\right)$. This simple formula shows a number of interesting trends: i) in an interferencelimited scenario, we observe an error-floor in the ABEP, which means that the ABEP does not go to zero as the AWGN goes to zero. This is because the single-user detector is interference-unaware; ii) the error-floor is lower (better performance) when either $N_{r}$ or the SIR increase; and iii) the error-floor is higher (worse performance) when $N_{t}$ increases. To overcome the error-floor, we need to increase either the transmit-energy $\left(E_{\xi}\right)$ of the probe link or the number of antennas, $N_{r}$, at the destination.

3) $N_{r} \gg 1$ : We have just remarked that multiple-access interference can be, in part, mitigated by adding more antennas at the receiver. We are interested in analyzing the asymptotic ABEP when $N_{r}$ is very large. To derive this result, we start from the last equality in the first line of (8). From [30, Eq. (2-1-136) and Eq. (2-1-139)], it follows that the $\mathrm{RV} \sqrt{\gamma_{\xi}}$ has mean and variance equal to $\mathrm{E}\left\{\sqrt{\gamma_{\xi}}\right\}=$ $2 \sigma_{\xi}\left[\Gamma\left(N_{r}+0.5\right) / \Gamma\left(N_{r}\right)\right]$ and $\mathrm{E}\left\{\left(\sqrt{\gamma_{\xi}}-\mathrm{E}\left\{\sqrt{\gamma_{\xi}}\right\}\right)^{2}\right\}=$ $4 \sigma_{\xi}^{2}\left\{N_{r}-\left[\Gamma\left(N_{r}+0.5\right) / \Gamma\left(N_{r}\right)\right]^{2}\right\}, \quad$ respectively. Since $\Gamma\left(N_{r}+0.5\right) / \Gamma\left(N_{r}\right) \cong \sqrt{N_{r}}$ if $N_{r} \gg 1$, then $\mathrm{E}\left\{\left(\sqrt{\gamma_{\xi}}-\mathrm{E}\left\{\sqrt{\gamma_{\xi}}\right\}\right)^{2}\right\} \rightarrow 0$ and $\mathrm{E}\left\{\sqrt{\gamma_{\xi}}\right\} \rightarrow 2 \sigma_{\xi} \sqrt{N_{r}}$. Thus, if $N_{r} \gg 1$, the $\mathrm{RV} \sqrt{\gamma_{\xi}}$ tends to a constant, i.e, $\sqrt{\gamma_{\xi}} \rightarrow 2 \sigma_{\xi} \sqrt{N_{r}}$, and, from (8), the ABEP reduces to $\mathrm{ABEP}_{\xi} \rightarrow\left(N_{t} / 2\right) Q\left(\sqrt{N_{r} \mathrm{SINR}}\right)$. We notice that the ABEP has a typical "waterfall" behavior and the effect of fading is drastically reduced. However, even though the AWGN is negligible with respect to multiple-access interference, we still have an error-floor. But it is much reduced. In particular, if $\mathrm{SNR}_{\xi} \gg 1$ and $\mathrm{SIR}=\mathrm{SNR}_{\xi} / \mathrm{INR}_{\backslash \xi} \gg 1$, then the number of receive-antenna $N_{r}^{*}$ that provide the target
$\mathrm{ABEP}_{\xi}^{*}$ is equal to $N_{r}^{*}=(1 / \mathrm{SIR})\left[Q^{-1}\left(2 \mathrm{ABEP}_{\xi}^{*} / N_{t}\right)\right]^{2}$. This is a very simple formula that can be used for a simple system design when the assumption $N_{r} \gg 1$ is reasonable.

\section{Single-USER Detection:}

\section{COMPARISON OF PSK, QAM, AND SSK MODULATION}

In this section, we aim at studying the performance of conventional QAM and PSK modulation, and at comparing them with SSK modulation. We consider a detector similar to (2), but the search space is given by the signal-constellation diagram rather than by the spatial-constellation diagram. Also, the methodology we use for performance analysis is similar to Section III. For this reason, and due to space constraints, we omit the details of the analytical derivation and report only the final results. Instead, we focus our attention on trying to understand advantages and disadvantages of using the spatialconstellation diagram as a source of information. Finally, we note that for QAM and PSK modulation the transmitter is equipped with a single-antenna, i.e., $N_{t}=1$.

Received signal and ML-optimum detector are shown in (10) on top of this page, where we have defined $D_{\xi}^{\left(s_{\xi}^{\left(y_{\xi}\right)}\right)}=$ $\sum_{r=1}^{N_{r}} \int_{T_{s}}\left|z^{(r)}(t)-\sqrt{E_{\xi}} h_{\xi}^{(r)} s_{\xi}^{\left(y_{\xi}\right)} w(t)\right|^{2} d t$, and have used a notation similar to (1) and (2). We emphasize that in (10) only the differences in the wireless channels are exploited to distinguish the users. Unlike, e.g., [21], no spreading sequences are used. This is in agreement with the SDMA/ChDMA multipleaccess schemes described in Section II.

The final expressions of the ABEP can be found in Table I, along with the asymptotic formulas that are valid for noiseand interference-limited scenarios. By comparing the results in Section III and Table I, the following general comments can be made: i) for QAM and PSK modulation the equality $\stackrel{(a)}{=}$ in (9) does not hold as the APEPs depend on the actual pair of points in the signal-constellation diagram. Thus, when this distance becomes small we can expect worse performance than SSK modulation; ii) for QAM we notice that the ABEP depends on the actual symbols transmitted by the interfering users. Thus, the signal-constellation diagram affects both the power of probe link and the aggregate interference; and iii) even though the asymptotic APEPs of QAM and PSK modulation in the noise-limited scenario look similar, we expect different results because the actual signal-constellation diagram is different.

Let us now study, in detail, whether/when we can expect that SSK modulation outperforms either QAM or PSK modulation. As a case study, we provide some formulas for PSK modulation, as its ABEP is simpler to manage. However, 
TABLE I

ABEP OF QAM AND PSK MODULATION. THE LAST FOUR ROWS SHOW FORMULAS RELATED TO THE ASYMPTOTIC ANALYSIS, SIMILAR TO SECTION III-A. A notation Similar to Section III is Adopted. $N_{H}\left(s_{\xi}^{\left(x_{\xi}\right)}, s_{\xi}^{\left(y_{\xi}\right)}\right)$ IS The Hamming DistanCE Between the Bit-TO-Symbol MAPping

$$
\text { OF } s_{\xi}^{\left(x_{\xi}\right)} \text { AND } s_{\xi}^{\left(y_{\xi}\right)} \text {. }
$$

\begin{tabular}{|c|c|}
\hline Modulation/Scenario & ABEP \\
\hline PSK (union-bound) & $\begin{array}{l}\operatorname{ABEP}_{\xi} \leq \frac{1}{M \log _{2}(M)} \sum_{x_{\xi}=1}^{M} \sum_{y_{\xi}=1}^{M} N_{H}\left(s_{\xi}^{\left(x_{\xi}\right)}, s_{\xi}^{\left(y_{\xi}\right)}\right) \underbrace{\operatorname{APEP}\left(s_{\xi}^{\left(x_{\xi}\right)} \rightarrow s_{\xi}^{\left(y_{\xi}\right)}\right)}_{\text {Eq. (8) }} \\
\mathrm{SINR}=\left[(1 / 2) \mathrm{SNR}_{\xi}\left|s_{\xi}^{\left(y_{\xi}\right)}-s_{\xi}^{\left(x_{\xi}\right)}\right|^{2}\right] /\left(1+\mathrm{INR}_{\backslash \xi}\right)\end{array}$ \\
\hline QAM (union-bound) & $\begin{array}{l}\operatorname{ABEP}_{\xi} \leq \frac{1}{M^{N_{u}} \log _{2}(M)} \sum_{x_{1}=1}^{M} \ldots \sum_{x_{\xi}=1}^{M} \ldots \sum_{x_{N_{u}}=1}^{M} \sum_{y_{\xi}=1}^{M} N_{H}\left(s_{\xi}^{\left(x_{\xi}\right)}, s_{\xi}^{\left(y_{\xi}\right)}\right) \underbrace{\operatorname{APEP}\left(s_{\xi}^{\left(x_{\xi}\right)} \rightarrow s_{\xi}^{\left(y_{\xi}\right)}\right)}_{\text {Eq. (8) }} \\
\mathrm{SINR}=\left[(1 / 2) \mathrm{SNR}_{\xi}\left|s_{\xi}^{\left(y_{\xi}\right)}-s_{\xi}^{\left(x_{\xi}\right)}\right|^{2}\right] /(1+\widetilde{\mathrm{INR}} \backslash \xi) \\
\widetilde{\mathrm{INR}}_{\backslash \xi}=\sum_{u \neq \xi=1}^{N_{u}}\left[\left(E_{u} \sigma_{u}^{2}\left|s_{u}^{\left(x_{u}\right)}\right|^{2}\right) / N_{0}\right]\end{array}$ \\
\hline PSK/QAM (noise-limited) & $\operatorname{APEP}\left(s_{\xi}^{\left(x_{\xi}\right)} \rightarrow s_{\xi}^{\left(y_{\xi}\right)}\right) \rightarrow\left(\begin{array}{c}2 N_{r}-1 \\
N_{r}\end{array}\right)\left|s_{\xi}^{\left(y_{\xi}\right)}-s_{\xi}^{\left(x_{\xi}\right)}\right|^{-2 N_{r}} \mathrm{SNR}_{\xi}^{-N_{r}}$ \\
\hline PSK (interference-limited) & $\operatorname{APEP}\left(s_{\xi}^{\left(x_{\xi}\right)} \rightarrow s_{\xi}^{\left(y_{\xi}\right)}\right) \rightarrow\left(\begin{array}{c}2 N_{r}-1 \\
N_{r}\end{array}\right)\left|s_{\xi}^{\left(y_{\xi}\right)}-s_{\xi}^{\left(x_{\xi}\right)}\right|^{-2 N_{r}}\left(\mathrm{SNR}_{\xi} / \mathrm{INR}_{\backslash \xi}\right)^{-N_{r}}$ \\
\hline QAM (interference-limited) & $\operatorname{APEP}\left(s_{\xi}^{\left(x_{\xi}\right)} \rightarrow s_{\xi}^{\left(y_{\xi}\right)}\right) \rightarrow\left(\begin{array}{c}2 N_{r}-1 \\
N_{r}\end{array}\right)\left|s_{\xi}^{\left(y_{\xi}\right)}-s_{\xi}^{\left(x_{\xi}\right)}\right|^{-2 N_{r}}\left(\mathrm{SNR}_{\xi} / \widetilde{\mathrm{INR}_{\backslash \xi}}\right)^{-N_{r}}$ \\
\hline
\end{tabular}

$$
\frac{\mathrm{ABEP}_{\xi}^{\mathrm{PSK}}}{\mathrm{ABEP}_{\xi}^{\mathrm{SSK}}} \rightarrow \frac{2}{N_{t}^{2} \log _{2}\left(N_{t}\right)} \sum_{x_{\xi}=1}^{N_{t}} \sum_{y_{\xi}=1}^{N_{t}}\left\{N_{H}\left(s_{\xi}^{\left(x_{\xi}\right)}, s_{\xi}^{\left(y_{\xi}\right)}\right)\left[2 /\left|s_{\xi}^{\left(y_{\xi}\right)}-s_{\xi}^{\left(x_{\xi}\right)}\right|^{2}\right]^{N_{r}}\right\}
$$

similar conclusions can be drawn for QAM as well. For a fair comparison, i.e., to guarantee the same bit rate, we assume $N_{t}=M$ and use the symbol $N_{t}$ in what follows. By using the formulas valid in the asymptotic regime, the ratio in (11) on top of this page can be computed, which holds for both noise- and interference-limited scenarios.

By looking into (11), the following conclusions can be made: i) SSK modulation will never be superior to PSK modulation if $\left|s_{\xi}^{\left(y_{\xi}\right)}-s_{\xi}^{\left(x_{\xi}\right)}\right|^{2} \geq 2$. This happens, e.g., when $N_{t}=M=2$ or $N_{t}=M=4$. On the other hand, when $N_{t}=M>4$ we can expect that a crossing point exists and that SSK modulation outperforms PSK modulation. In other words, the higher the target bit rate is, the more advantageous SSK modulation is. This conclusion holds for QAM as well. This result was argued by simulation in [2] for a noise-limited scenario, but no proof was given. Also, we have shown that the trend holds in the presence of multiple-access interference as well; ii) for those setups where SSK outperforms QAM/PSK modulation we have $\left|s_{\xi}^{\left(y_{\xi}\right)}-s_{\xi}^{\left(x_{\xi}\right)}\right|^{2}<2$ for some signal-constellation points. In this case, the ratio in (11) increases exponentially with $N_{r}$ : the larger $N_{r}$ is, the higher the performance gain provided by SSK modulation is. Also this result was argued by simulation in [2] for a noise-limited scenario, but no proof was given. We have shown that the trend holds in the presence of multiple-access interference as well; and iii) by direct inspection of (11), we can compute the asymptotic SNR and SIR gains of a modulation scheme with respect to the other as $\Delta_{\mathrm{SNR}}=$ $\Delta_{\mathrm{SIR}}=\left(10 / N_{r}\right) \log _{10}\left(\mathrm{ABEP}_{\xi}^{\mathrm{PSK}} / \mathrm{ABEP}_{\xi}^{\mathrm{SSK}}\right)$.

\section{GSSK Modulation with Single-USER Detection}

The working principle of GSSK modulation is as follows [8]: i) each user encodes blocks of $\left|\log _{2}\left(\begin{array}{c}N_{t} \\ N_{\mathrm{ta}}\end{array}\right)\right|$ bits into a point of a spatial-constellation diagram of size $N=$ $2^{\left\lfloor\log _{2}\left(\begin{array}{c}N_{t} \\ N_{\mathrm{ta}}\end{array}\right)\right\rfloor}$, which enables $N_{\text {ta }}$ antennas to be switched on for data transmission while all the other antennas are kept silent, and ii) similar to SSK modulation, the receiver solves a $N$-hypothesis testing problem to estimate the $N_{\text {ta }}$ antennas that are not idle, which results in the estimation of the message emitted by the encoder of the probe link.

Similar to Section III, received signal and ML-optimum detector are given in (12) on top of the next page, where $D_{\xi}^{\left(\mathbf{y}_{\xi}^{(a)}\right)}=$ $\sum_{r=1}^{N_{r}} \int_{T_{s}}\left|z^{(r)}(t)-\sum_{a=1}^{N_{\mathrm{ta}}}\left[\sqrt{\left(E_{\xi} / N_{\mathrm{ta}}\right)} h_{\xi}^{\left(y_{\xi}^{(a)}, r\right)} w(t)\right]\right|^{2} d t$ 


$$
\left\{\begin{array}{l}
z^{(r)}(t)=\sum_{a=1}^{N_{\mathrm{ta}}}\left[\sqrt{\frac{E_{\xi}}{N_{\mathrm{ta}}}} h_{\xi}^{\left(x_{\xi}^{(a)}, r\right)} w(t)\right]+\sum_{u \neq \xi=1}^{N_{u}} \sum_{a=1}^{N_{\mathrm{ta}}}\left[\sqrt{\frac{E_{u}}{N_{\mathrm{ta}}}} h_{u}^{\left(x_{u}^{(a)}, r\right)} w(t)\right]+n^{(r)}(t) \\
\hat{\mathbf{x}}_{\xi}=\underset{\mathbf{y}_{\xi} \in \Theta_{\mathrm{GSSK}}}{\arg \min }\left\{D_{\xi}^{\left(\mathbf{y}_{\xi}^{(a)}\right)}\right\}=\underset{\mathbf{y}_{\xi} \in \Theta_{\mathrm{GSSK}}}{\arg \min }\left\{\sum_{r=1}^{N_{r}} \int_{T_{s}}\left|z^{(r)}(t)-\sum_{a=1}^{N_{\mathrm{ta}}}\left[\sqrt{\frac{E_{\xi}}{N_{\mathrm{ta}}}} h_{\xi}^{\left(y_{\xi}^{(a)}, r\right)} w(t)\right]\right|^{2} d t\right\}
\end{array}\right.
$$

$$
\left\{\begin{array}{l}
\operatorname{ABEP}_{\xi} \leq \frac{1}{N \log _{2}(N)} \sum_{\mathbf{x}_{\xi} \in \Theta_{\mathrm{GSSK}}} \sum_{\mathbf{y}_{\xi} \in \Theta_{\mathrm{GSSK}}} N_{H}\left(\mathbf{x}_{\xi}, \mathbf{y}_{\xi}\right) \underbrace{\operatorname{APEP}\left(\mathbf{x}_{\xi} \rightarrow \mathbf{y}_{\xi}\right)}_{\mathrm{Eq} .} \\
\mathrm{SINR}=\frac{1}{2} \frac{\mathrm{SNR}_{\xi}}{1+\mathrm{INR}_{\backslash \xi}} \frac{N_{\mathrm{ta}}^{\neq}\left(\mathbf{x}_{\xi}, \mathbf{y}_{\xi}\right)}{N_{\mathrm{ta}}}
\end{array}\right.
$$

$\mathbf{x}_{\xi}=\left[x_{\xi}^{(1)}, x_{\xi}^{(2)}, \ldots, x_{\xi}^{\left(N_{\mathrm{ta}}\right)}\right]$ denotes the $N_{\mathrm{ta}}$-dimensional vector of active antenna-indexes of the probe link; $\hat{\mathbf{x}}_{\xi}$ is its ML-optimum estimate; $\mathbf{y}_{\xi}$ is the trial instance of $\mathbf{x}_{\xi}$ used in the hypothesis-testing problem; and $\Theta_{\mathrm{GSSK}}$ is the spatial-constellation diagram of GSSK modulation, i.e., the set of $N=2^{\left\lfloor\log _{2}\left(\begin{array}{c}N_{t} \\ N_{\mathrm{ta}}\end{array}\right)\right\rfloor}$ vectors of antenna-indexes that can possibly be activated for transmission. $\Theta_{\text {GSSK }}$ is a subset of the set of all possible combinations, i.e., $\left(\begin{array}{c}N_{t} \\ N_{\mathrm{ta}}\end{array}\right)$, of antennaindexes. Furthermore, we notice that in (12) the available energy per transmission of each user, $E_{u}$, is scaled by $N_{\text {ta }}$ to ensure a fair comparison with SSK modulation. In particular, a uniform power-allocation strategy is assumed among the antennas. Improvement is possible by using opportunistic power allocation [31], but it is not here considered.

The ABEP can be computed by using an analytical derivation similar to SSK modulation. The main difference consists in taking into account that, for each user, the equivalent channel seen by the intended receiver is the summation of the channels originated from $N_{\text {ta }}$ antennas. Accordingly, by comparing (1) and (12) we notice that the signal models become the same if we replace $h_{u}^{\left(x_{u}, r\right)}$ in (1) with $\left(1 / \sqrt{N_{\mathrm{ta}}}\right) \sum_{a=1}^{N_{\mathrm{ta}}} h_{u}^{\left(x_{u}^{(a)}, r\right)}$ in (12) for $u=1,2, \ldots, N_{u}$. Thus, with the help of the formal substitution $h_{u}^{\left(x_{u}, r\right)} \mapsto\left(1 / \sqrt{N_{\mathrm{ta}}}\right) \sum_{a=1}^{N_{\mathrm{ta}}} h_{u}^{\left(x_{u}^{(a)}, r\right)}$, all the analytical steps in (4)-(8) can formally be repeated. By doing so, the only modification turns out to be the final expression of $\gamma_{\xi}$ in (8), which can be generalized as $\gamma_{\xi}=$

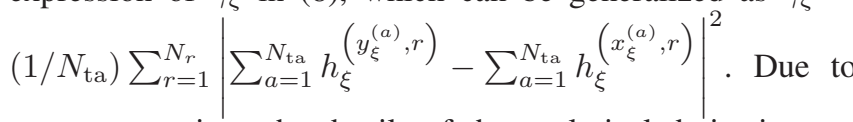
space constraints, the details of the analytical derivation are here omitted. On the other hand, we focus our attention on analyzing advantages and disadvantages of SSK and GSSK modulations.

The final expression of the ABEP is summarized in (13) on top of this page, where $N_{H}\left(\mathbf{x}_{\xi}, \mathbf{y}_{\xi}\right)$ is the Hamming distance between the bit-to-antenna-index-tuple mapping of $\mathbf{x}_{\xi}$ and $\mathbf{y}_{\xi} ; N_{\mathrm{ta}}^{\neq}\left(\mathbf{x}_{\xi}, \mathbf{y}_{\xi}\right)$ is the number of non-shared antenna-indexes of $\mathbf{x}_{\xi}$ and $\mathbf{y}_{\xi}$. For example, if $\mathbf{x}_{\xi}=[1,2,3]$ and $\mathbf{y}_{\xi}=$ $[1,3,4]$, then $N_{\mathrm{ta}}^{\neq}\left(\mathbf{x}_{\xi}, \mathbf{y}_{\xi}\right)=2$, i.e., indexes 2 and 4. Let us here emphasize that $N_{\mathrm{ta}}^{\neq}\left(\mathbf{x}_{\xi}, \mathbf{y}_{\xi}\right)$ comes from the generalized expression of $\gamma_{\xi}$ given above; and the other symbols have the same definition as in Section III. Furthermore, if $N_{\mathrm{ta}}=1$,
GSSK reduces to SSK modulation. In this case, $N=N_{t}$ and $N_{\mathrm{ta}}^{\neq}\left(\mathbf{x}_{\xi}, \mathbf{y}_{\xi}\right)=2$ for each pair $\left(\mathbf{x}_{\xi}, \mathbf{y}_{\xi}\right) \in \Theta_{\mathrm{GSSK}} \times \Theta_{\mathrm{GSSK}}$, and, as expected, (13) reduces to (9). Finally, by direct inspection and whatever the effective spatial-constellation diagram is, the inequalities $2 \leq N_{\text {ta }}^{\neq}\left(\mathbf{x}_{\xi}, \mathbf{y}_{\xi}\right) \leq 2 N_{\text {ta }}$ hold. The lowerbound corresponds to SSK modulation, while the upper-bound corresponds to a spatial-constellation diagram where all the indexes in $\mathbf{x}_{\xi}$ and $\mathbf{y}_{\xi}$ are different. This result is exploited in Section V-A to compare SSK and GSSK modulations. Finally, we note that, unlike SSK modulation, in GSSK modulation the equality $\stackrel{(a)}{=}$ in (9) does not hold because the PEPs actually depend on $\left(\mathbf{x}_{\xi}, \mathbf{y}_{\xi}\right)$, even for i.i.d. fading channels.

\section{A. Asymptotic Analysis and Comparison with SSK Modulation}

Similar to SSK modulation, we can analyze the performance in noise- and interference-limited scenarios. In particular, the APEP in (13) reduces to $\operatorname{APEP}\left(\mathbf{x}_{\xi} \rightarrow \mathbf{y}_{\xi}\right) \rightarrow$ $\left[N_{\mathrm{ta}} / N_{\mathrm{ta}}^{\neq}\left(\mathbf{x}_{\xi}, \mathbf{y}_{\xi}\right)\right]^{N_{r}}\left(\begin{array}{c}2 N_{r}-1 \\ N_{r}\end{array}\right) \Upsilon^{-N_{r}}$, where $\Upsilon=\mathrm{SNR}_{\xi}$ in a noise-limited scenario and $\Upsilon=$ SIR in an interferencelimited scenario, respectively. Accordingly, from Section III-A we can compute the following ratio:

$$
\frac{\operatorname{APEP}^{\mathrm{GSSK}}\left(\mathbf{x}_{\xi} \rightarrow \mathbf{y}_{\xi}\right)}{\operatorname{APEP}_{\xi}^{\mathrm{SSK}}} \rightarrow\left[\frac{2 N_{\mathrm{ta}}}{N_{\mathrm{ta}}^{\neq}\left(\mathbf{x}_{\xi}, \mathbf{y}_{\xi}\right)}\right]^{N_{r}}
$$

From (14), the following conclusions can be drawn: i) since $2 \leq N_{\mathrm{ta}}^{\neq}\left(\mathbf{x}_{\xi}, \mathbf{y}_{\xi}\right) \leq 2 N_{\mathrm{ta}}$, it follows that $\operatorname{APEP}^{\mathrm{GSSK}}\left(\mathbf{x}_{\xi} \rightarrow \mathbf{y}_{\xi}\right) \geq \overline{\operatorname{APEP}}_{\xi}^{\mathrm{SSK}}$, i.e., GSSK modulation is always worse than $\mathrm{SSK}^{\xi}$ modulation, regardless of the actual spatial-constellation diagram; and ii) the extra SNR or SIR that we need for GSSK to get the same APEP as for SSK modulation is $\Delta_{\Upsilon}=\left(10 / N_{r}\right) \log _{10}\left(\operatorname{APEP}^{\mathrm{GSSK}}\left(\mathbf{x}_{\xi} \rightarrow \mathbf{y}_{\xi}\right) / \operatorname{APEP}_{\xi}^{\mathrm{SSK}}\right)$, which lies in the interval $0 \leq \Delta_{\Upsilon} \leq 10 \log _{10}\left(N_{\mathrm{ta}}\right)$. This result is very important because it shows that the larger the number, $N_{\text {ta }}$, of active antennas is, the worse GSSK modulation with respect to SSK modulation is. The intuitive reason for this trend is as follows. If $N_{\mathrm{ta}}^{\neq}\left(\mathbf{x}_{\xi}, \mathbf{y}_{\xi}\right)<2 N_{\mathrm{ta}}$, it means that $\mathbf{x}_{\xi}$ and $\mathbf{y}_{\xi}$ have some antenna-indexes in common, which cancel out in the hypothesis testing problem. Since the transmit-energy is distributed among the active antennas, this results in a destructive interference cancelation effect: 


$$
\hat{\mathbf{x}}=\underset{\substack{\mathbf{y}=\left[y_{1}, y_{2}, \ldots, y_{N_{u}}\right] \\ \text { for } y_{u}=1,2, \ldots, N_{t}}}{\arg \min }\left\{D^{(\mathbf{y})}\right\}=\underset{\substack{\mathbf{y}=\left[y_{1}, y_{2}, \ldots y_{N_{u}}\right] \\ \text { for } y_{u}=1,2, \ldots, N_{t}}}{\arg \min }\left\{\sum_{r=1}^{N_{r}} \int_{T_{s}}\left|z^{(r)}(t)-\sum_{u=1}^{N_{u}}\left[\sqrt{E_{u}} h_{u}^{\left(y_{u}, r\right)} w(t)\right]\right|^{2} d t\right\}
$$

$$
\left\{\begin{array}{l}
\operatorname{ABEP}_{\xi} \stackrel{(a)}{\leq} \frac{1}{N_{t}^{N_{u}}} \sum_{x_{1}=1}^{N_{t}} \cdots \sum_{x_{N_{u}}=1}^{N_{t}} \sum_{y_{1}=1}^{N_{t}} \cdots \sum_{y_{N_{u}}=1}^{N_{t}}\left[\frac{N_{H}\left(x_{\xi}, y_{\xi}\right)}{\log _{2}\left(N_{t}\right)} \operatorname{APEP}_{x_{\xi} \neq y_{\xi}}(\mathbf{x} \rightarrow \mathbf{y})\right] \\
\operatorname{APEP}_{x_{\xi} \neq y_{\xi}}(\mathbf{x} \rightarrow \mathbf{y}) \stackrel{(b)}{=}\left(1-\delta_{x_{\xi}, y_{\xi}}\right) \mathrm{E}_{\mathbf{h}}\{\operatorname{Pr}\{\mathbf{x} \neq \mathbf{y}\}\} \stackrel{(c)}{=}\left(1-\delta_{x_{\xi}, y_{\xi}}\right) \mathrm{E}_{\mathbf{h}}\{\operatorname{PEP}(\mathbf{x} \rightarrow \mathbf{y})\}
\end{array}\right.
$$

$$
\mathrm{E}_{\mathbf{h}}\{\operatorname{PEP}(\mathbf{x} \rightarrow \mathbf{y})\}=\left[\frac{1}{2}\left(1-\sqrt{\frac{\mathrm{AggrSNR}}{2+\operatorname{AggrSNR}}}\right)\right]^{N_{r}} \sum_{r=1}^{N_{r}}\left\{\left(\begin{array}{c}
N_{r}-1+r \\
r
\end{array}\right)\left[\frac{1}{2}\left(1+\sqrt{\frac{\operatorname{AggrSNR}}{2+\operatorname{AggrSNR}}}\right)\right]^{r}\right\}
$$

we transmit power on the common indexes, but it does not contribute to the ML-optimum decision process. Thus, for better performance we should keep the number of active antennas as small as possible. Finally, we emphasize that the conclusions in i) and ii) hold for the ABEP too, as it can be derived from (9) and (13).

\section{Vi. Multi-User Detection: ANALYSIS AND COMPARISON}

In the sections above, we have studied the ABEP when the receiver is interference-unaware and exploits, for data detection, only the CSI of the probe link. The main advantage of this receiver is the low computational complexity, while its main disadvantage is the error-floor when multiple-access interference is the dominant effect. In this section, we study the performance of the ML-optimum joint multi-user detector [21], which exploits CSI of all the active users, and, thus, is interference-aware. We compute accurate union-bound estimates of the ABEP for all the modulation schemes (SSK, GSSK, PSK, and QAM) of interest, and, through asymptotic analysis, we provide some weaker bounds to better understand the behavior of the detector. Due to space constraints, we provide a detailed derivation of the ABEP of SSK modulation, and give only the final result for the other modulation schemes. Finally, we emphasize that with respect to, e.g., [21] our study does not exploit any signature code for multiple-access capabilities, but exploits only the differences/randomness of the CIRs among the active users. This agrees with the definition of SDMA/ChDMA multiple-access schemes described in Section II.

Let us consider SSK modulation. The received signal is always given by (1), but the detector is different. In particular, the ML-optimum joint multi-user detector is given in (15) on top of this page [21], [27], where the following notation is used: i) $\mathbf{x}=\left[x_{1}, x_{2}, \ldots x_{N_{u}}\right]$ is the vector of antenna-indexes that is actually active in the considered time-slot; ii) $\hat{\mathbf{x}}=\left[\hat{x}_{1}, \hat{x}_{2}, \ldots \hat{x}_{N_{u}}\right]$ is its ML-optimum estimate; iii) $\mathbf{y}=\left[y_{1}, y_{2}, \ldots y_{N_{u}}\right]$ is the trial instance of $\hat{\mathbf{x}}$ used in the hypothesis-testing problem; and iv) $D^{(\mathbf{y})}=$ $\sum_{r=1}^{N_{r}} \int_{T_{s}}\left|z^{(r)}(t)-\sum_{u=1}^{N_{u}}\left[\sqrt{E_{u}} h_{u}^{\left(y_{u}, r\right)} w(t)\right]\right|^{2} d t$.
Using arguments similar to (3), the ABEP of a generic user, e.g., $\xi$, can be upper-bounded as shown in (16) on top of this page, where: i) in $\stackrel{(a)}{\leq}$, the scaling factor $1 / N_{t}^{N_{u}}$ takes into account that the $N_{t}^{N_{u}}$ possible vectors $\mathrm{x}$ are equiprobable; ii) in $\stackrel{(a)}{\leq}, N_{H}\left(x_{\xi}, y_{\xi}\right) / \log _{2}\left(N_{t}\right)$ accounts for the percentage of wrong bits between $x_{\xi}$ and $y_{\xi}$, which is related to bit-toantenna-index mapping. It is worth mentioning that, as far as user $\xi$ is concerned, an error occurs if and only if $x_{\xi} \neq y_{\xi}$. In other words, even though $\mathbf{x} \neq \mathbf{y}$, this does not imply that we have an error for all the $N_{u}$ users. In the best case, and error occurs for one user only; iii) in $\stackrel{(a)}{\leq}$, the ABEP is conditioned upon the event $x_{\xi} \neq y_{\xi}$ to take into account that we are interested in computing the ABEP of user $\xi$; iv) in $\stackrel{(b)}{=}$, the factor $\left(1-\delta_{x_{\xi}, y_{\xi}}\right)$ takes into account that, as mentioned above, there is no contribution to the ABEP if $x_{\xi}=y_{\xi}$, even though $\mathbf{x} \neq \mathbf{y}$; and $\mathrm{v}) \stackrel{(c)}{=}$ tells us that the ABEP is uniquely determined by the PEPs of the pair $(\mathbf{x}, \mathbf{y})$, i.e., $\operatorname{PEP}(\mathbf{x} \rightarrow \mathbf{y})$.

The PEP, PEP $(\mathbf{x} \rightarrow \mathbf{y})$, conditioned upon all the fading channel gains, can be computed by using analytical steps similar to Section III. The final result is as follows:

$$
\operatorname{PEP}(\mathbf{x} \rightarrow \mathbf{y})=Q\left(\sqrt{\frac{1}{4 N_{0}} \sum_{r=1}^{N_{r}}\left|\sum_{u=1}^{N_{u}} \sqrt{E_{u}}\left(h_{u}^{\left(y_{u}, r\right)}-h_{u}^{\left(x_{u}, r\right)}\right)\right|^{2}}\right)
$$

Finally, by exploiting, similar to (8), the properties of ChiSquare RVs, we can remove the conditioning over all fading channel statistics. After some algebra, and using [30, Eq. (2-1136), Eq. (2-1-137)] and [30, Eq. (14-4-14), Eq. (14-4-15)], we can obtain (18) on top of this page, where AggrSNR = $\sum_{u=1}^{N_{u}}\left\{\left[E_{u} \sigma_{u}^{2}\left(1-\delta_{x_{u}, y_{u}}\right)\right] / N_{0}\right\}$ is the Aggregate SNR. We note that the delta function, $\delta_{x_{u}, y_{u}}$, in AggrSNR takes into account that if $x_{u}=y_{u}$, then $h_{u}^{\left(y_{u}, r\right)}-h_{u}^{\left(x_{u}, r\right)}=0$ in (17) and, thus, it does not contribute to the SNR seen by the detector. In other words, the antenna-indexes shared by $\mathbf{x}$ and $\mathbf{y}$ cancel out in the hypothesis-testing problem.

By comparing (8) and (18), we notice, as expected, that the main difference between single- and multi-user detector is the absence of error-floor for high SNRs in (18), i.e., $\mathrm{E}_{\mathbf{h}}\{\operatorname{PEP}(\mathbf{x} \rightarrow \mathbf{y})\} \rightarrow 0$ if $N_{0} \rightarrow 0$. The price to be paid 
TABLE II

ABEP OF PSK, QAM, AND GSSK MODULATIONS WITH MULTI-USER DETECTION. FOR GSSK MODULATION, WE HAVE EXPLICITLY USED THE IDENTITY $N=2^{\left\lfloor\log _{2}\left(\begin{array}{c}N_{t} \\ N_{\mathrm{ta}}\end{array}\right)\right\rfloor}$, AND $\mathbf{x}_{1: N_{u}}=\left[\mathbf{x}_{1}, \mathbf{x}_{2}, \ldots, \mathbf{x}_{N_{u}}\right]$ IS USED AS A SHORT-HAND FOR "VeCTORS OF VECTORS". FURTHERMORE, $s^{(\mathbf{x})}=\left[s_{1}^{\left(x_{1}\right)}, s_{2}^{\left(x_{2}\right)}, \ldots, s_{N_{u}}^{\left(x_{N_{u}}\right)}\right]$.

\begin{tabular}{|c|c|c|}
\hline Modulation & \multicolumn{2}{|c|}{ ABEP } \\
\hline PSK/QAM & $\begin{array}{l}\operatorname{ABEP}_{\xi} \leq \frac{1}{M^{N_{u}}} \sum_{x_{1}=1}^{M} \ldots \sum_{x_{N_{u}}=1}^{M} \sum_{y_{1}=1}^{M} \ldots \sum_{y_{N_{u}}=1}^{M} \\
\operatorname{AggrSNR}=\sum_{u=1}^{N_{u}}\left[\frac{E_{u} \sigma_{u}^{2}}{2 N_{0}}\left|s_{\xi}^{\left(y_{\xi}\right)}-s_{\xi}^{\left(x_{\xi}\right)}\right|^{2}\right]\end{array}$ & {$[\frac{N_{H}\left(s_{\xi}^{\left(x_{\xi}\right)}, s_{\xi}^{\left(y_{\xi}\right)}\right)}{\log _{2}(M)} \underbrace{\operatorname{APEP}_{x_{\xi} \neq y_{\xi}}\left(s^{(\mathbf{x})} \rightarrow s^{(\mathbf{y})}\right)}_{\text {Eq. (18), Eq. (20) }}]$} \\
\hline GSSK & $\begin{array}{l}\operatorname{ABEP}_{\xi} \leq \frac{1}{2^{N_{u}}\left[\log _{2}\left(\begin{array}{c}N_{t} \\
N_{\mathrm{ta}}\end{array}\right)\right]} \sum_{\mathbf{x}_{1} \in \Theta_{\mathrm{GSSK}}} \cdots \sum_{\mathbf{x}_{N_{u}} \in \Theta_{\mathrm{GSSK}} \mathbf{y}} \\
\overline{\operatorname{APEP}}_{\mathbf{x}_{\xi} \neq \mathbf{y}_{\xi}}\left(\mathbf{x}_{1: N_{u}} \rightarrow \mathbf{y}_{1: N_{u}}\right)=\frac{N_{H}\left(\mathbf{x}_{\xi}, \mathbf{y}_{\xi}\right)}{\left[\log _{2}\left(\begin{array}{c}N_{t} \\
N_{\mathrm{ta}}\end{array}\right)\right]} \underbrace{\operatorname{APEP} \mathbf{x}} \\
\operatorname{AggrSNR}=\sum_{u=1}^{N_{u}}\left[\frac{E_{u} \sigma_{u}^{2}}{2 N_{0}} \frac{N_{\mathrm{ta}}^{\neq}\left(x_{u}, y_{u}\right)}{N_{\mathrm{ta}}}\left(1-\delta_{x_{u}, y_{u}}\right)\right]\end{array}$ & $\begin{array}{l}\sum_{\in \Theta_{\mathrm{GSSK}}} \cdots \sum_{\mathbf{y}_{N_{u}} \in \Theta_{\mathrm{GSSK}}} \overline{\operatorname{APEP}}_{\mathbf{x}_{\xi} \neq \mathbf{y}_{\xi}}\left(\mathbf{x}_{1: N_{u}} \rightarrow\right. \\
; \neq \mathbf{y}_{\xi}\left(\mathbf{x}_{1: N_{u}} \rightarrow \mathbf{y}_{1: N_{u}}\right)\end{array}$ \\
\hline
\end{tabular}

$$
\operatorname{ABEP}_{\xi} \rightarrow\left[N_{t}^{N_{u}} \log _{2}\left(N_{t}\right)\right]^{-1}\left(\begin{array}{c}
2 N_{r}-1, \\
N_{r}
\end{array}\right) 2^{-N_{r}} \sum_{\mathbf{x}} \sum_{\mathbf{y}}\left[\left(1-\delta_{x_{\xi}, y_{\xi}}\right) N_{H}\left(x_{\xi}, y_{\xi}\right) \operatorname{AggrSNR}^{-N_{r}}\right]
$$

$$
\Delta_{\mathrm{SNR}_{\xi}} \rightarrow \frac{10}{N_{r}} \log _{10}\left(\frac{\mathrm{ABEP}_{\xi}}{\mathrm{ABEP}_{\xi}^{\mathrm{SULB}}}\right)=\frac{10}{N_{r}} \log _{10}\left(\frac{1}{N_{t}^{\left(N_{u}+1\right)} \log _{2}\left(N_{t}\right)} \sum_{\mathbf{x}} \sum_{\mathbf{y}}\left[\frac{\left(1-\delta_{x_{\xi}, y_{\xi}}\right) N_{H}\left(x_{\xi}, y_{\xi}\right) E_{\xi} \sigma_{\xi}^{2}}{2 \sum_{u=1}^{N_{u}}\left[E_{u} \sigma_{u}^{2}\left(1-\delta_{x_{u}, y_{u}}\right)\right]}\right]\right)
$$

for this performance improvement is a higher computational complexity, as the detector in (15) has a complexity that increases exponentially with $N_{u}$. By using a similar analytical derivation, Table II summarizes the ABEP of PSK, QAM, and GSSK modulations. The performance comparison is postponed to Section VIII for various system setups. As far as QAM and PSK modulation are concerned, it is worth mentioning that: i) formally, the ABEP of both modulation schemes is the same, but the signal-constellation diagram is different; and ii) thanks to the assumptions of synchronous multiple-access interference and SDMA/ChDMA multiple-access scheme, the framework in Table II is useful to model spatial-multiplexing MIMO systems with ML-optimum detection as well. More specifically, in this case $N_{u}$ streams are simultaneously transmitted by a single user equipped with $N_{t}=N_{u}$ antennas [32]. The only difference is that in spatial-multiplexing MIMO we are interested in the average ABEP among all the streams, i.e. $\mathrm{ABEP}=\left(1 / N_{u}\right) \sum_{u=1}^{N_{u}} \mathrm{ABEP}_{u}$, which can be derived from Table II. Some simulation results about spatial-multiplexing MIMO schemes are given in Section VIII.

\section{A. SSK Modulation: Asymptotic Analysis and Bounds}

In this section, we study the asymptotic ABEP and compute some bounds to shed lights on the perfor- mance of multi-user detectors for SSK modulation. For ease of notation, in what follows we use the short-hand $\sum_{x_{1}=1}^{N_{t}} \cdots \sum_{x_{N_{u}}=1}^{N_{t}} \sum_{y_{1}=1}^{N_{t}} \cdots \sum_{y_{N_{u}}=1}^{N_{t}}(\cdot) \mapsto \sum_{\mathbf{x}} \sum_{\mathbf{y}}(\cdot)$.

1) AggrSNR $\gg 1$ : Similar to Section III-A, for high SNR the ABEP in (16) is asymptotically equal to (19) on top of this page. If $N_{u}=1$, it can be shown that the ABEP (henceforth called Single-User-Lower-Bound (SULB), as it provides the performance without multiple-access interference) reduces to $\mathrm{ABEP} \mathrm{SULB}_{\xi}^{\mathrm{SULB}} \rightarrow 2^{-\left(N_{r}+1\right)}\left(\begin{array}{c}2 N_{r}-1 \\ N_{r}\end{array}\right) N_{t} \mathrm{SNR}_{\xi}^{-N_{r}}$. As expected, $\mathrm{ABEP}_{\xi}^{\mathrm{SULB}}$ is equivalent to the ABEP of the single-user detector computed in Section III-A for the noise-limited scenario. It is interesting to understand the relation between the ABEP of the multi-user detector and the SULB. By direct inspection, the extra SNR needed in a multi-user scenario to achieve the same ABEP as in a noise-limited scenario is given in (20) on top of this page. The formula in (20) provides a quite accurate estimate of the extra SNR to get the same $\mathrm{ABEP}$ as in a noise-limited environment. However, it is not much insightful because it explicitly depends on the bit-toantenna-index mapping. To deeper understand, we analyze some special cases and provide some weaker bounds, which better reveal the behavior of multi-user detection for SSK modulation. 


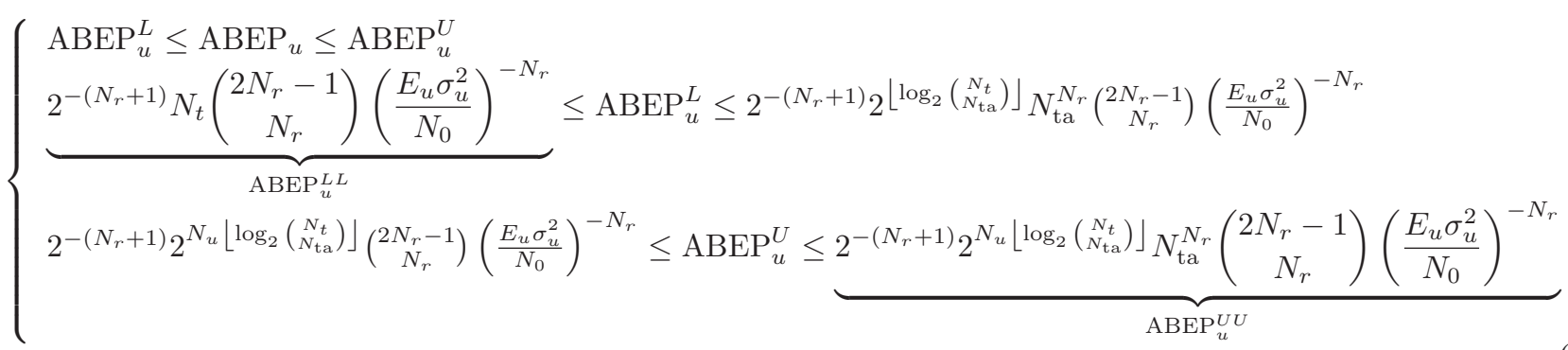

2) $E_{w} \sigma_{w}^{2} \ll E_{u} \sigma_{u}^{2} \forall u=1,2, \ldots N_{u}$ (strong interference scenario): Let us assume that among the $N_{u}$ users there is a user, henceforth called "worst" $(w)$ user, with the worst propagation channel. The ABEP of this user can be readily estimated with arguments similar to [21], as the user experiences very strong aggregate interference from the remaining $N_{u}-1$ users. In this case, it is known that the multi-user detector can perfectly estimate and reduce to zero the interference generated by the other users. In this case, its ABEP tends to the SULB, i.e., $\operatorname{ABEP}_{w} \rightarrow 2^{-\left(N_{r}+1\right)} N_{t}\left(\begin{array}{c}2 N_{r}-1 \\ N_{r}\end{array}\right)\left[\left(E_{w} \sigma_{w}^{2}\right) / N_{0}\right]^{-N_{r}}$.

3) $E_{b} \sigma_{b}^{2} \gg E_{u} \sigma_{u}^{2} \forall u=1,2, \ldots N_{u}$ (weak interference scenario): Let us assume that among the $N_{u}$ users there is a user, henceforth called "best" (b) user, with the best propagation channel. This scenario is more complicated to study than the strong interference case. However, we provide a tight bound to estimate the ABEP. If $E_{b} \sigma_{b}^{2} \gg$ $E_{u} \sigma_{u}^{2} \forall u=1,2, \ldots N_{u}$, then from (18) we have AggrSNR $\rightarrow$ $\left(E_{b} \sigma_{b}^{2}\right) / N_{0}$. Accordingly, for high SNR we get:

$$
\begin{aligned}
\operatorname{ABEP}_{b} & \rightarrow \frac{1}{N_{t}^{N_{u}} \log _{2}\left(N_{t}\right)}\left(\begin{array}{c}
2 N_{r}-1 \\
N_{r}
\end{array}\right)\left[\frac{2 E_{b} \sigma_{b}^{2}}{N_{0}}\right]^{-N_{r}} \\
& \times \sum_{\mathbf{x}} \sum_{\mathbf{y}} N_{H}\left(x_{\xi}, y_{\xi}\right)
\end{aligned}
$$

By direct inspection, it is easy to show that $\sum_{\mathbf{x}} \sum_{\mathbf{y}} N_{H}\left(x_{\xi}, y_{\xi}\right)=N_{t}^{\left(N_{u}-1\right)} N_{t}^{\left(N_{u}-1\right)}\left(N_{t}^{2} / 2\right) \log _{2}\left(N_{t}\right)$. Accordingly, (21) simplifies to $\mathrm{ABEP}_{b} \rightarrow$ $2^{-\left(N_{r}+1\right)} N_{t}^{N_{u}}\left(\begin{array}{c}2 N_{r}-1 \\ N_{r}\end{array}\right)\left[\left(E_{b} \sigma_{b}^{2}\right) / N_{0}\right]^{-N_{r}}$, which is a very simple and easy-to-compute formula. Thus, the SNR gap with respect to the SULB can be computed as $\Delta_{\mathrm{SNR}_{b}}=\left(10 / N_{r}\right) \log _{10}\left(\mathrm{ABEP}_{b} / \mathrm{ABEP}_{b}^{\mathrm{SULB}}\right)=$ $10\left[\left(N_{u}-1\right) / N_{r}\right] \log _{10}\left(N_{t}\right)$. This formula is very insightful, as it provides a simple relation among all the parameters of interests, and, so, can be used for a quick system design. For example, for a given $\Delta_{\mathrm{SNR}_{b}}, N_{t}$ and $N_{r}$, we can compute the maximum number of users that can share the wireless medium to guarantee the desired ABEP. Also, we notice that the larger $N_{r}$ is, the smaller $\Delta_{\mathrm{SNR}_{b}}$ is, and the ABEP turns out to be very close to the SULB.

4) Generic user (arbitrary interference scenario): The analysis of the ABEP for a generic user can accurately be performed by using (16) and (18), or by using the asymptotic result for AggrSNR $\gg 1$. However, its performance can be easily lower- and upper-bounded as $\operatorname{ABEP}_{u}^{L} \leq \mathrm{ABEP}_{u} \leq$

$$
\begin{aligned}
& \operatorname{ABEP}_{u}^{U}: \\
& \left\{\begin{array}{l}
\operatorname{ABEP}_{u}^{L}=2^{-\left(N_{r}+1\right)} N_{t}\left(\begin{array}{c}
2 N_{r}-1 \\
N_{r}
\end{array}\right)\left(\frac{E_{u} \sigma_{u}^{2}}{N_{0}}\right)^{-N_{r}} \\
\operatorname{ABEP}_{u}^{U}=2^{-\left(N_{r}+1\right)} N_{t}^{N_{u}}\left(\begin{array}{c}
2 N_{r}-1 \\
N_{r}
\end{array}\right)\left(\frac{E_{u} \sigma_{u}^{2}}{N_{0}}\right)^{-N_{r}}
\end{array}\right.
\end{aligned}
$$

The lower-bound, $\mathrm{ABEP}_{u}^{L}$, comes from the fact that the ABEP cannot the better than the SULB. On the other hand, the upper-bound, $\operatorname{ABEP}_{u}^{U}$, comes from the fact that the ABEP cannot be worse than the scenario with weak interference, which is the worst-case situation for any user. The SNR gap between the two bounds is $\Delta_{\mathrm{SNR}_{u}}=\left(10 / N_{r}\right) \log _{10}\left(\mathrm{ABEP}_{u}^{U} / \mathrm{ABEP}_{u}^{L}\right)=$ $10\left[\left(N_{u}-1\right) / N_{r}\right] \log _{10}\left(N_{t}\right)$. We notice that, for any user, the larger $N_{r}$ is, the closer to the SULB the ABEP is. While, the larger $N_{u}$ or $N_{t}$ (i.e., the rate) are, the further from the SULB the ABEP is. These trends are reasonable, will be validated by simulation in Section VIII, and can be exploited for a simple design of very general and complicated MIMO systems.

\section{B. GSSK Modulation: Asymptotic Analysis and Bounds}

As far as GSSK modulation is concerned, we can perform a similar asymptotic analysis. In particular, insightful bounds can be obtained by combining the study in Section V-A and in Section VI-A. More specifically, for a generic user, the ABEP is lower- and upper-bounded as shown in (23) on top of this page. In particular, $\mathrm{ABEP}_{u}^{L}$ corresponds to the SULB of GSSK modulation. It actually depends on the number, $N_{\mathrm{ta}}^{\neq}\left(\mathbf{x}_{\xi}, \mathbf{y}_{\xi}\right)$, of non-shared antenna-indexes of $\mathbf{x}_{\xi}$ and $\mathbf{y}_{\xi}$. However, in Section V-A we have proved that $N_{\mathrm{ta}}^{\neq}\left(\mathbf{x}_{\xi}, \mathbf{y}_{\xi}\right)$ can be lower-bounded by the SULB of SSK modulation, i.e., $\operatorname{ABEP}_{u}^{L L}$ in (23), as well as upper-bounded by considering the worst-case scenario with $N_{\mathrm{ta}}^{\neq}\left(\mathbf{x}_{\xi}, \mathbf{y}_{\xi}\right)=2$ for every $\mathbf{x}_{\xi}$ and $\mathbf{y}_{\xi}$, as given in the right hand-side of (23). On the other hand, $\operatorname{ABEP}_{u}^{U}$ corresponds, similar to Section VI-A, to the worst-case scenario with weak interference. Its lowerand upper-bound shown in (23) can be obtained by setting $N_{\text {ta }}^{\neq}\left(\mathbf{x}_{\xi}, \mathbf{y}_{\xi}\right) / N_{\text {ta }}=2$ and $N_{\text {ta }}^{\neq}\left(\mathbf{x}_{\xi}, \mathbf{y}_{\xi}\right) / N_{\text {ta }}=2 / N_{\text {ta }}$ for every $\mathbf{x}_{\xi}$ and $\mathbf{y}_{\xi}$, respectively. In fact, in Section $\mathrm{V}$ we have shown that $2 \leq N_{\mathrm{ta}}^{\neq}\left(\mathbf{x}_{\xi}, \mathbf{y}_{\xi}\right) \leq 2 N_{\mathrm{ta}}$ for every $\mathbf{x}_{\xi}$ and $\mathbf{y}_{\xi}$.

Overall, from (23) we conclude that the ABEP of GSSK modulation lies in the interval $\mathrm{ABEP}_{u}^{L L} \leq \mathrm{ABEP} \leq$ $\mathrm{ABEP}_{u}^{U U}$. More specifically, the SNR gap is $\Delta_{\mathrm{SNR}_{u}}=$ $\left(10 / N_{r}\right) \log _{10}\left(\operatorname{ABEP}_{u}^{U U} / \mathrm{ABEP}_{u}^{L L}\right)=10 \log _{10}\left(N_{t}\right)+$ 
$\left(10 / N_{r}\right) \log _{10}\left(2^{N_{u}\left\lfloor\log _{2}\left(N_{t}, N_{\mathrm{ta}}\right)\right\rfloor} / N_{t}\right)$. Also in this case, $\Delta_{\mathrm{SNR}_{u}}$ provides reasonable and insightful outcomes about the behavior of GSSK modulation. Finally, we emphasize that $\operatorname{ABEP}_{u}^{L L}$ is the SULB of SSK modulation, and, so, we can readily estimate the relation among the two modulation schemes for generic MIMO systems.

\section{Coordinated Multi-Point (CoMP) SPACE MOdulation}

In the previous sections, we have shown that exploiting the spatial-constellation diagram can be beneficial to improve the performance. However, to better exploit the spatialconstellation diagram and have a substantial performance improvement without sacrificing the bit rate, large antennaarrays are needed. While for some emerging transmission frequency bands this might not be a problem, as many antennas can efficiently be packed in a device [19], [20], in general there might be physical limitations on the number of antennas of each array. Two solutions to overcome this problem are, e.g., GSSK modulation [8] and SM [6]. GSSK modulation enables a better exploitation of the available antennas at the transmitter by activating more antennas and increasing the bit rate. However, in Section V we have seen that, in general, the ABEP of GSSK modulation is worse than SSK modulation. SM is a hybrid modulation scheme where spatial- and signalconstellation diagrams are jointly exploited to find a good trade-off between bit rate and performance. However, for small antenna-arrays the benefit of the spatial-constellation diagram can be exploited only in part, as many information bits need to be encoded into the signal-constellation diagram. Also, Generalized SM (GSM) might be another solution that combines GSSK modulation and SM for a better trade-off.

In this section, we wish to bring to the attention of the reader that another way of providing large antenna-arrays in SSK modulation is to exploit the concept of virtual MIMO [33], also known as Distributed Antenna System (DAS), BS cooperation, or CoMP transmission [34]-[38]. The main idea is to share the antenna-arrays of multiple transmitters, thus having a larger equivalent (virtual) antenna-array that can be used to encode a larger number of information bits. The basic idea is the following. Let $N_{t}^{\mathrm{BS}}$ be the number of BSs connected to, e.g., a Base Station Controller (BSC) via a reliable wired backhaul link, such that all of them can receive the message that the core network is intended to transmit to a remote handset. Also, let $N_{t}^{\mathrm{AR}}$ be the number of transmit-antennas available in each BS. Accordingly, the virtual MIMO system of $N_{t}^{\mathrm{BS}}$ BSs has a total number of $N_{t}=N_{t}^{\mathrm{BS}} N_{t}^{\mathrm{AR}}$ antennas that form a virtual (distributed and very large) spatial-constellation diagram. With this approach, CoMP-SSK modulation can transmit $\log _{2}\left(N_{t}\right)=\log _{2}\left(N_{t}^{\mathrm{BS}}\right)+\log _{2}\left(N_{t}^{\mathrm{AR}}\right)$ bits per time-slot. Hybrid solutions such as CoMP-GSSK, CoMP-SM, and CoMP-GSM are possible with their own advantages and disadvantages. A comprehensive study of all these solutions is out of the scope of this paper. In this paper, we are only interested in putting forth the concept of CoMP-SSK modulation as a practical way of achieving very large bit rates by exploiting just the spatial-constellation diagram. Also, we wish to understand the asymptotic performance gain of SSK modulation as $N_{t}$ increases without bound. In Section VIII, we will show various results for, e.g., $N_{t}>64$, which might not be achievable, in practice, with a single BS, but can be obtained by resorting to a CoMP approach (e.g., with $\left(N_{t}^{\mathrm{BS}}, N_{t}^{\mathrm{AR}}\right)=(4,16)$ or $\left.\left(N_{t}^{\mathrm{BS}}, N_{t}^{\mathrm{AR}}\right)=(8,8)\right)$.

With respect to conventional BS cooperation methods [36], in CoMP-SSK modulation the backhaul has less stringent requirements as the coordinated BSs do not have to exchange data for cooperative beamforming, but the backhaul is used only for disseminating the information from the core network to the BSs. Furthermore, we emphasize that since the cooperative BSs do not perform distributed beamforming, no transmitCSI is required, even though it might be beneficial [31].

\section{Numerical and Simulation Results}

In this section, we provide some numerical results to compare the performance of various modulation schemes in the presence of multiple-access interference, and to substantiate our analytical findings. In particular, the system model introduced and described in Section II is accurately reproduced in our simulation environment, and various MIMO setups and interference scenarios are analyzed. The specific simulation parameters can be found in the caption of each figure. The results are obtained by assuming $E_{u}=E_{m} \forall u=$ $1,2, \ldots, N_{u}$. So, the near-far effect is modeled through the fading parameters $\sigma_{u}^{2}$.

In Fig. 1, we observe the near-far effect for two MIMO setups with a different number of receive-antennas. As computed analytically in Section III, the ABEP gets worse as the interference increases. Also, the system is more robust to multiple-access interference as $N_{r}$ increases. We notice that our analytical model (union-bound) is very accurate. Only when $\mathrm{ABEP} \geq 10^{-1}$, it starts being less accurate. This is a reasonable outcome as the union-bound is tight only for low values of ABEP, while the interference introduces an error-floor. However, the model can, in general, well track the error-floor, as predicted in Section III. In Fig. 2, we study the robustness of single-user detection to the number of active users $N_{u}$. As predicted by the union-bound in Section III, the ABEP gets worse when increasing $N_{u}$, but the receiver can work quite well when $N_{r} \geq 3$. We notice that the error-floor increases with $N_{u}$. In Fig. 3 and Fig. 4, we compare the performance of SSK, GSSK, QAM, and PSK modulations for various target bit rates. We remind the reader that for large values of $N_{t}$, SSK modulation is implemented through the CoMP approach. As predicted in Section IV and Section V, QAM and PSK modulation outperform SSK modulation only if the bit rate if less than $2 \mathrm{bits} / \mathrm{s} / \mathrm{Hz}$, and SSK modulation always outperforms GSSK modulation. Also, the higher the target bit rate is, the larger the gap is. This confirms the findings in Section IV, and highlights that using the spatialconstellation diagram is beneficial with and without multipleaccess interference. Overall the bounds can very well capture the behavior of all the modulation schemes if the error floor is not too high. This means that they are useful for all scenarios of practical interest. In Fig. 5 and in Fig. 6, we 

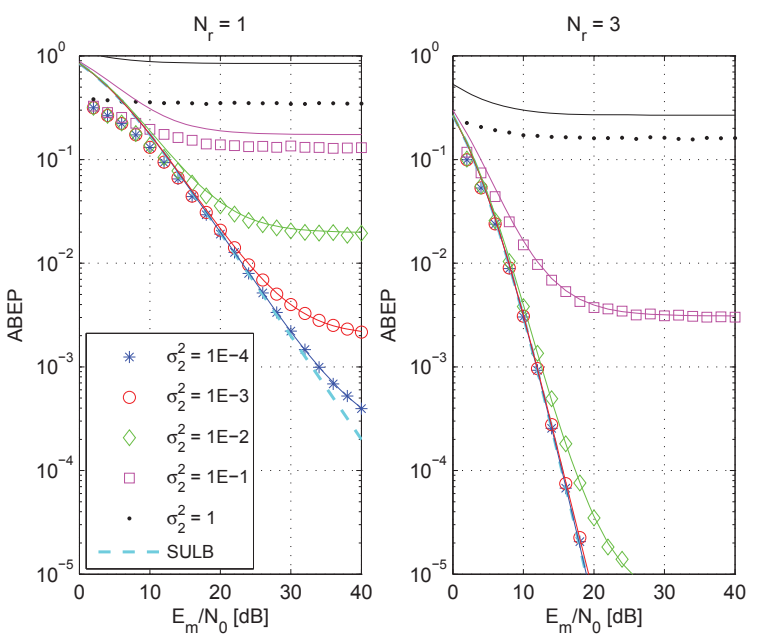

Fig. 1. ABEP of SSK modulation with single-user detection. Setup: $N_{t}=8$; $N_{u}=2 ; \sigma_{1}^{2}=1 ; N_{r}=1$ (left) and $N_{r}=3$ (right). Markers show Monte Carlo simulations and solid lines the analytical model (i.e., (8) and (9)). The ABEP of user 1 (probe/intended link) is shown. SULB stands for SingleUser Lower Bound, i.e., it represents the scenario with no multiple-access interference.

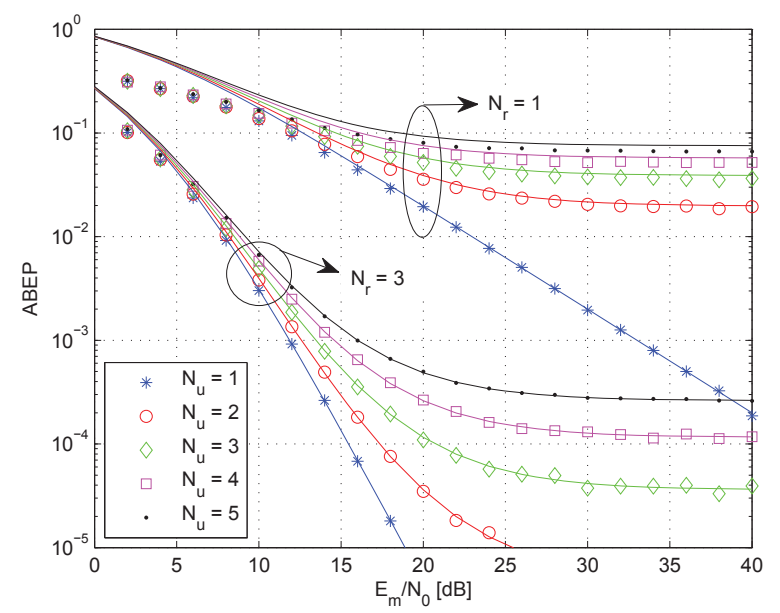

Fig. 2. ABEP of SSK modulation with single-user detection. Setup: $N_{t}=8$ $\sigma_{1}^{2}=1 ; \sigma_{i}^{2}=10^{-2}$ for $i=2,3, \ldots, N_{u} ; N_{r}=1$ and $N_{r}=3$. Markers show Monte Carlo simulations and solid lines the analytical model (i.e., (8) and (9)). The ABEP of user 1 (probe/intended link) is shown.

analyze the performance of SSK modulation with respect to the number of receive-antennas. If $N_{r}=3$ (Fig. 5), we observe a non-negligible performance gain, if the bit rate if greater than 2 bits $/ \mathrm{s} / \mathrm{Hz}$, provided by SSK modulation with respect to QAM. The price to be paid is, of course, the need to exploit the CoMP principle to achieve very high bit rates, e.g., when $N_{t}=64$. However, the SNR gain is so significant to motivate the CoMP approach. On the other hand, if $N_{r}=1$ (Fig. 6), we notice that QAM is always superior to SSK modulation, while SSK modulation is better than PSK and GSSK modulations. This result is not available in the literature even for $N_{u}=1$, as the vast majority of papers typically consider MIMO setups with $N_{r}>1$. As a consequence, if a single-user scenario is considered and the
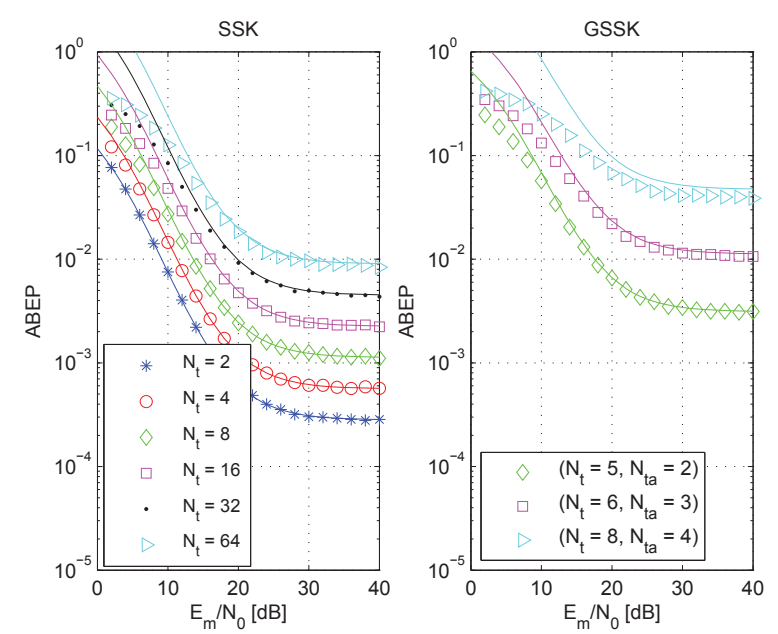

Fig. 3. ABEP of SSK (left) and GSSK (right) modulations with singleuser detection. Setup: $N_{u}=3 ; \sigma_{1}^{2}=1 ; \sigma_{i}^{2}=10^{-2}$ for $i=2,3, \ldots, N_{u}$; $N_{r}=2$. Markers show Monte Carlo simulations and solid lines the analytical model (i.e., (8) and (9) for SSK modulation and (13) for GSSK modulation). The ABEP of user 1 (probe/intended link) is shown.
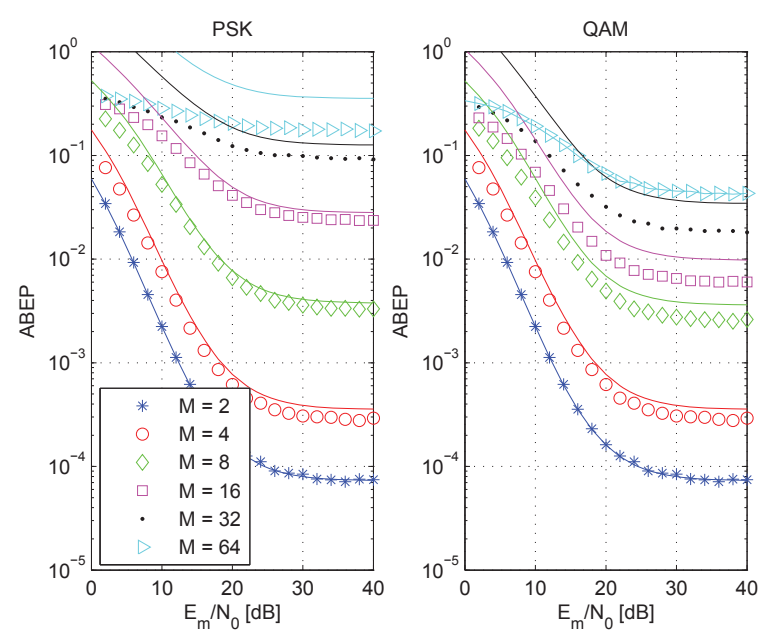

Fig. 4. ABEP of PSK (left) and QAM (right) modulations with singleuser detection. Setup: $N_{u}=3 ; \sigma_{1}^{2}=1 ; \sigma_{i}^{2}=10^{-2}$ for $i=2,3, \ldots, N_{u}$; $N_{r}=2$. Markers show Monte Carlo simulations and solid lines the analytical model (i.e., the union-bound in the first and second row of Table I). The ABEP of user 1 (probe/intended link) is shown.

receiver can be equipped with only one receive-antenna, then SSK modulation is not the best choice and we should use QAM. In all the other cases, SSK modulation is superior to QAM. Furthermore, in a multi-user scenario we can very unlikely design and use a MIMO system with $N_{r}=1$, since, as shown in Fig. 6 for $N_{u}=2$, the ABEP rapidly gets worse with the target bit rate. In scenarios with multiuser interference and single-user detection we are forced to increase $N_{r}$ to get adequate performance. In these situations, SSK modulation is always better than QAM. Finally, in Fig. 7 we study the ABEP for very high bit rates (CoMP-SSK has a large number of cooperative BSs). We can observe a significant performance gain of SSK modulation with respect to all the other modulation schemes. Also, GSSK modulation 

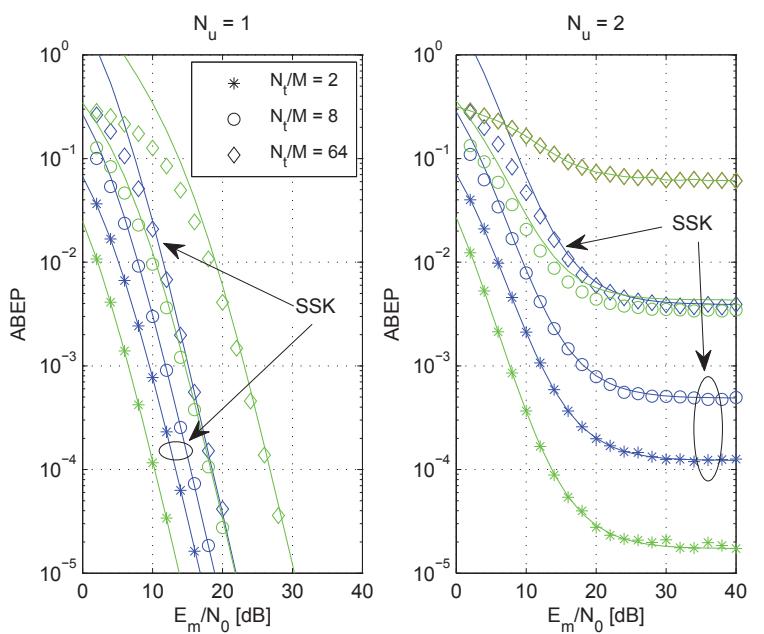

Fig. 5. ABEP of SSK (blue curves) and QAM (green curves) modulations with single-user detection. Setup: $N_{u}=1$ (left) and $N_{u}=2$ (right); $\sigma_{1}^{2}=1$ and $\sigma_{2}^{2}=5 \times 10^{-2} ; N_{r}=3$. Markers show Monte Carlo simulations and solid lines the analytical model (i.e., (8) and (9) for SSK modulation and the union-bound in the second row of Table I). The ABEP of user 1 (probe/intended link) is shown.
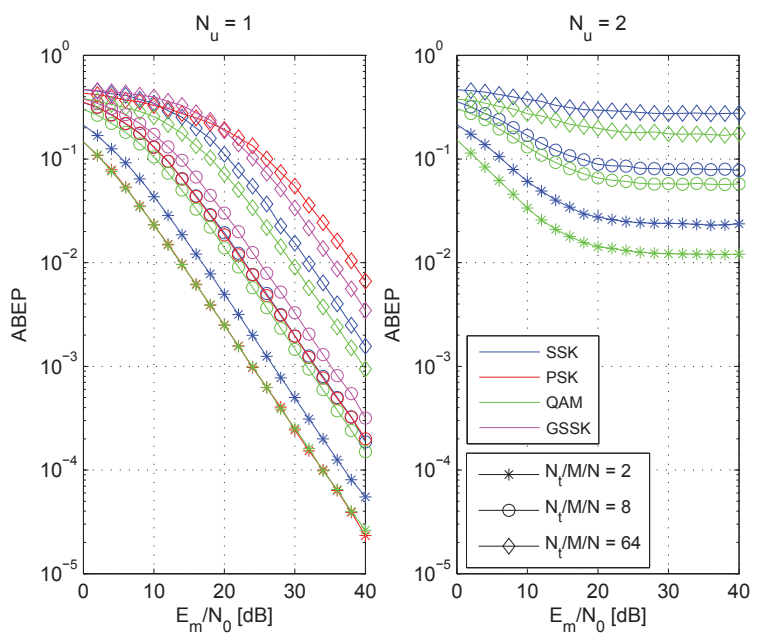

Fig. 6. ABEP of SSK (blue curves), GSSK (magenta curves), PSK (red curves), and QAM (green curves) modulations with single-user detection. Setup: $N_{u}=1$ (left) and $N_{u}=2$ (right); $\sigma_{1}^{2}=1$ and $\sigma_{2}^{2}=5 \times 10^{-2}$; $N_{r}=1$. Only Monte Carlo simulations (markers plus solid lines) are shown for ease of readability. The ABEP of user 1 (probe/intended link) is shown.

significantly outperforms QAM. Overall, our analysis and simulations confirm the potential benefits of using the spatialconstellation diagram in both single- and multi-user scenarios.

In Figs. 3, 4, 7, we have compared the ABEP of SSK/GSSK modulations and single-antenna PSK/QAM with the main goal of understanding the performance gap among these transmission technologies when ML-optimum performance can be achieved with low-complexity single-stream decoding at the receiver. In other words, the receiver has almost the same complexity for all the modulation schemes. However, when considering the complexity of the transmitter, the comparison in Figs. 3, 4, 7 might appear a bit unfair for single-antenna PSK/QAM
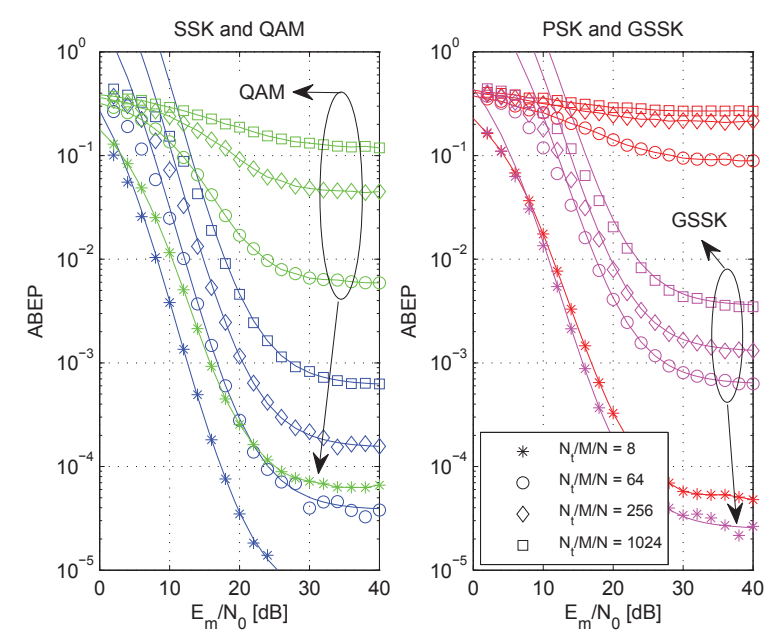

Fig. 7. ABEP of, on the left, SSK (blue curves) and QAM (green curves), and, on the right, PSK (red curves) and GSSK (magenta curves) modulations with single-user detection. Setup: $N_{u}=2 ; \sigma_{1}^{2}=1$ and $\sigma_{2}^{2}=10^{-2} ; N_{r}=3$. For GSSK modulation we have: $\left(N_{t}, N_{\mathrm{ta}}\right)=(5,2)$ if $N=8 ;\left(N_{t}, N_{\mathrm{ta}}\right)=$ $(8,4)$ if $N=64 ;\left(N_{t}, N_{\mathrm{ta}}\right)=(11,4)$ if $N=256$; and $\left(N_{t}, N_{\mathrm{ta}}\right)=$ $(12,5)$ if $N=1024$. For SSK and GSSK modulations, markers show Monte Carlo simulations and solid lines the analytical model (i.e., (8) and (9) for SSK modulation and (13) for GSSK modulation). For QAM and PSK modulations, only Monte Carlo simulations (markers plus solid lines) are shown for ease of readability. The ABEP of user 1 (probe/intended link) is shown.

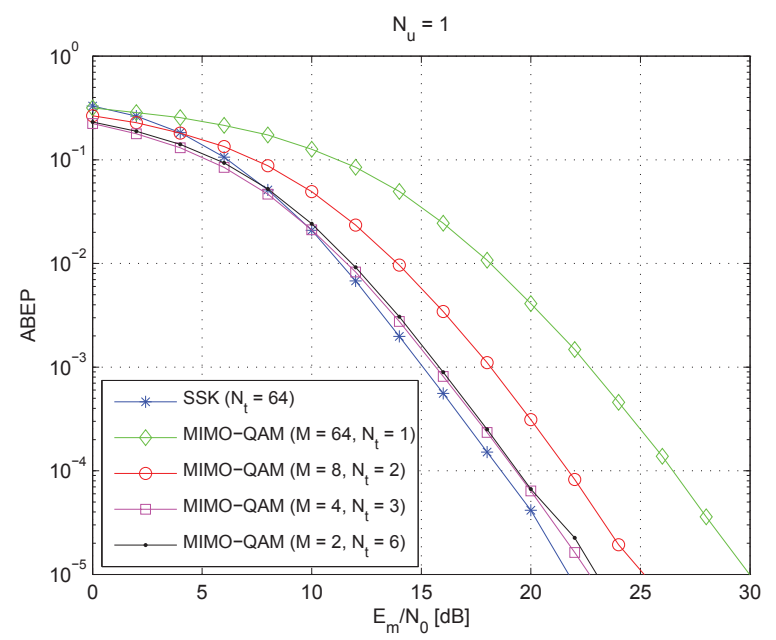

Fig. 8. ABEP of SSK modulation and spatial-multiplexing MIMO with QAM (MIMO-QAM) for $N_{u}=1$. A single-stream and a multi-stream decoders are used at the receiver for SSK modulation and spatial-multiplexing MIMO, respectively. Setup: $\sigma^{2}=1$ for all the wireless links; $N_{r}=3$. Only Monte Carlo simulations are shown. The curves of SSK modulation with $N_{t}=64$, and MIMO-QAM with $N_{t}=1$ and $M=64$ are the same as those shown in Fig. 5.

systems, as SSK/GSSK modulations need large antenna-arrays to achieve the same transmission rate. Motivated by this consideration, in Fig. 8 we study a complementary situation in which PSK/QAM systems using spatial-multiplexing MIMO $[39$, Sec. I-A] are compared to SSK/GSSK modulations. In this case, PSK/QAM systems need a multi-stream decoder to guarantee ML-optimum performance. More specifically, in a point-to-point link the detector is the same as in Section VI, with the only exception that all the streams are simul- 
taneously transmitted from the same device under the total transmit-power constraint. Unlike Figs. 3, 4, 7, in this case the comparison is certainly unfair for SSK/GSSK modulations, which need much less decoding complexity for ML-optimum performance. However, we feel important to show this setup and to analyze all the possibilities. For simplicity, we limit the study to the setup with $N_{u}=1$, since ML-optimum decoding for spatial-multiplexing MIMO in the presence of multiple-access interference would require a two-fold multistream decoder to cope with inner (i.e., due to multiplexing many streams at the same transmitter) and outer (i.e., due to the multi-user scenario) interferences. This scenario would require a more practical and sub-optimal decoder, e.g., based on sphere-decoding [40], to keep the complexity at a reasonable level. Accordingly, this study is postponed to future research. From Fig. 8, important considerations can be made. We notice that by increasing the number of antennas at the transmitter, spatial-multiplexing MIMO with QAM achieves, as expected, better performance than single-antenna QAM. However, the price to pay for this performance improvement is, among the others, multi-stream decoding at the receiver. Very interestingly, we see that SSK modulation is never worse than spatialmultiplexing MIMO, even though SSK modulation needs just low-complexity single-user decoding. In this case the price to pay is the need to have multiple radiating elements at the transmitter, even though just one of them is active and, thus, no multiple transmit-chains are needed. Similar to [6] and [7], these results clearly show the potential advantages of using the spatial-constellation diagram when comparing SSK modulation to more complicated MIMO schemes. However, it should be emphasized that, because of the very different hardware and computational complexity requirements, no conclusive statements can be made about the superiority of one transmission technology against the other. The only pragmatic conclusion that can be drawn is the clear potential gain that might come from using the spatial-constellation diagram, along with the inherent performance/complexity trade-off among different modulation schemes.

In Figs. 9-17, we show that ABEP with multi-user detection. The setup is the same as in Figs. 1-7. So, the interested reader can readily compare single- and multi-user detection for the same operating conditions. In Fig. 9 and Fig. 10, we study the accuracy of the lower- and upper-bound derived in Section VI-A. We observe that, for various strong/weak interference scenarios, the bounds well track the behavior of the system. In particular, Fig. 10 shows that the ABEP of a generic user that is subject to neither strong nor weak interference is well bounded by our analytical frameworks. For those users where the assumption of strong/weak interference can be made, the bounds are asymptotically-tight. Figure 11 clearly shows that no error floor is present with multi-user detection, and the ABEP goes to zero if the noise is very small. This is an important result and the confirmation that both modulation and multiple-access can be guaranteed by exploiting only the randomness of the wireless channels.

Similar to Fig. 3 and Fig. 4, in Figs. 12-15 we compare the performance of various modulation schemes. Also in this case, we notice the non-negligible performance gain of

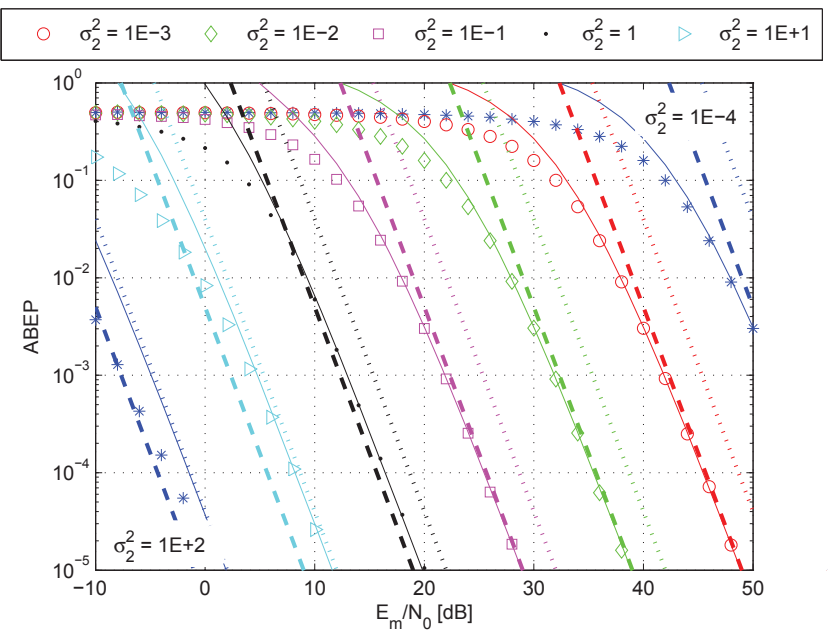

Fig. 9. ABEP of SSK modulation with multi-user detection. Setup: $N_{t}=8$; $N_{u}=2 ; \sigma_{1}^{2}=1 ; N_{r}=3$. Markers show Monte Carlo simulations and solid lines the analytical model (i.e., (16) and (18)). Furthermore, dashed lines show the estimated lower-bound (i.e., $\operatorname{ABEP}_{u}^{L}$ in (22)), which corresponds to the Single-User Lower-Bound (SULB) when no multiple-access interference is present; and dotted lines show the estimated upper-bound (i.e., $\operatorname{ABEP}_{u}^{U}$ in (22)). The ABEP of user 2 (probe/intended link) is shown.

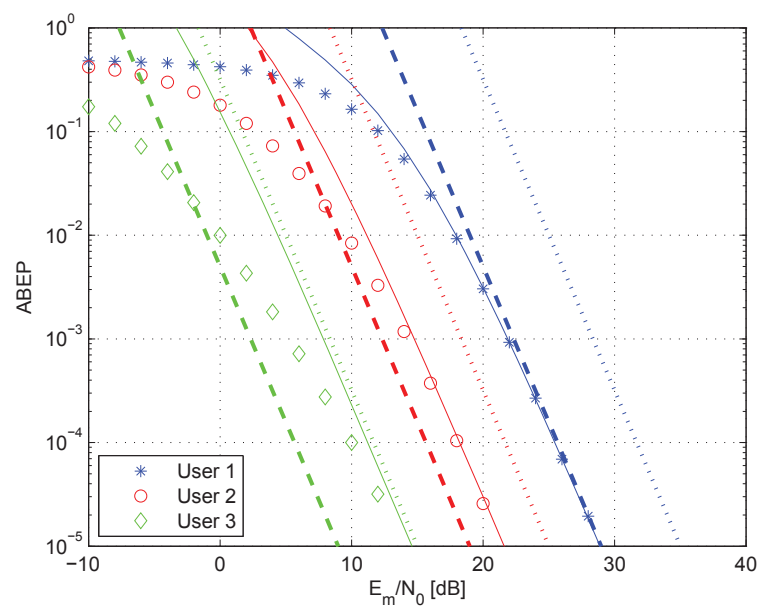

Fig. 10. ABEP of SSK modulation with multi-user detection. Setup: $N_{t}=8$; $N_{u}=3 ; \sigma_{1}^{2}=0.1, \sigma_{2}^{2}=1, \sigma_{3}^{2}=10 ; N_{r}=3$. Markers show Monte Carlo simulations and solid lines the analytical model (i.e., (16) and (18)). Furthermore, dashed lines show the estimated lower-bound (i.e., $\operatorname{ABEP}_{u}^{L}$ in (22)), which corresponds to the Single-User Lower-Bound (SULB) when no multiple-access interference is present; and dotted lines show the estimated upper-bound (i.e., $\operatorname{ABEP}_{u}^{U}$ in (22)). The ABEP of all the users is shown.

SSK modulation. Overall, the predictions in Section VI are confirmed, and the bounds developed in Section VI-A and Section VI-B agree with Monte Carlo simulations. A very interesting result is shown in Fig. 16. Unlike Fig. 6, we observe that, for a bit rate grater than 2 bits $/ \mathrm{s} / \mathrm{Hz}$, with multi-user detection SSK modulation is not worse than QAM even if $N_{r}=1$. In particular, we observe a crossing point for high SNRs, where the ABEP of SSK modulation is at least the same as QAM. This result clearly highlights that SSK modulation with multi-user detection is inherently more robust than QAM to multiple-access interference. Finally, Fig. 17 shows a result 


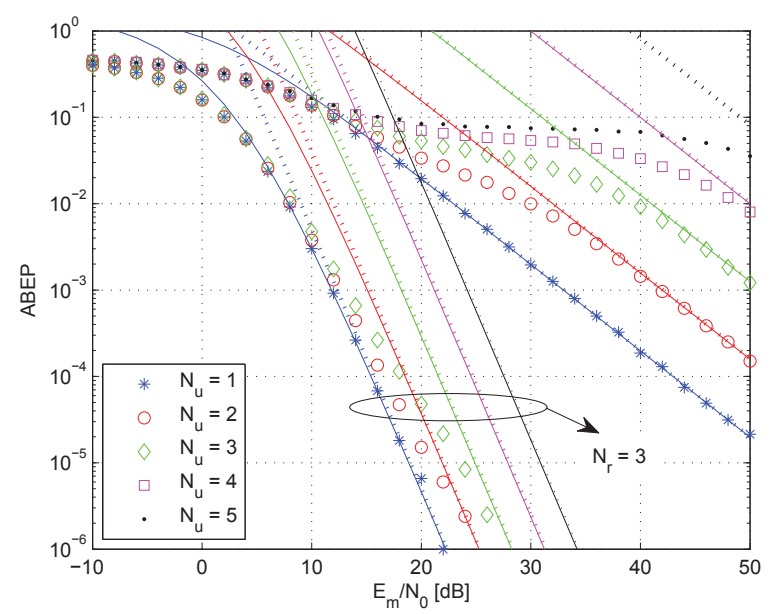

Fig. 11. ABEP of SSK modulation with multi-user detection. Setup: $N_{t}=8$; $\sigma_{1}^{2}=1$ and $\sigma_{i}^{2}=10^{-2}$ for $i=2,3, \ldots, N_{u} ; N_{r}=1$ and $N_{r}=3$. Markers show Monte Carlo simulations and solid lines the analytical model (i.e., (16) and (18)). Furthermore, dotted lines show the estimated upperbound (i.e., $\mathrm{ABEP}_{u}^{U}$ in (22)). The ABEP of user 1 (probe/intended link) is shown. It is worth mentioning that some simulation results (markers) are not shown due to the long simulation time for medium/high values of $E_{m} / N_{0}$.

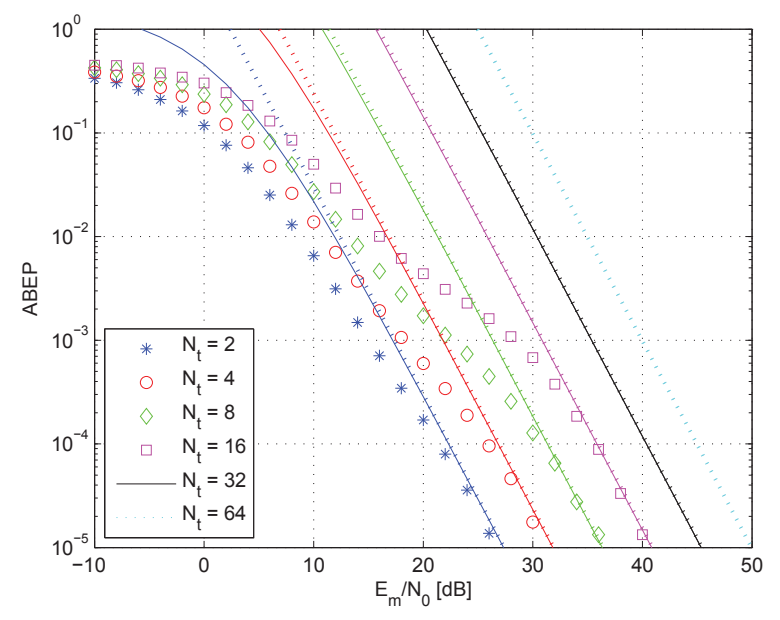

Fig. 12. ABEP of SSK modulation with multi-user detection. Setup: $N_{u}=$ $3 ; \sigma_{1}^{2}=1$ and $\sigma_{i}^{2}=10^{-2}$ for $i=2,3, \ldots, N_{u} ; N_{r}=2$. Markers show Monte Carlo simulations and solid lines the analytical model (i.e., (16) and (18)). Furthermore, dotted lines show the estimated upper-bound (i.e., $\mathrm{ABEP}_{u}^{U}$ in (22)). The ABEP of user 1 (probe/intended link) is shown. It is worth mentioning that some simulation results (markers) are not shown due to the long simulation time for medium/high values of $E_{m} / N_{0}$.

similar to Fig. 7 for high bit rates. The performance gain of SSK modulation is well confirmed in this case too.

\section{CONCLUSION}

In this paper, we have proposed a comprehensive framework to study the ABEP of SSK/GSSK modulations over Rayleigh fading channels with multiple-access interference. The frameworks are useful for single- and multi-user detectors. Furthermore, simple upper- and lower-bounds have been developed, and have been used to get insightful information

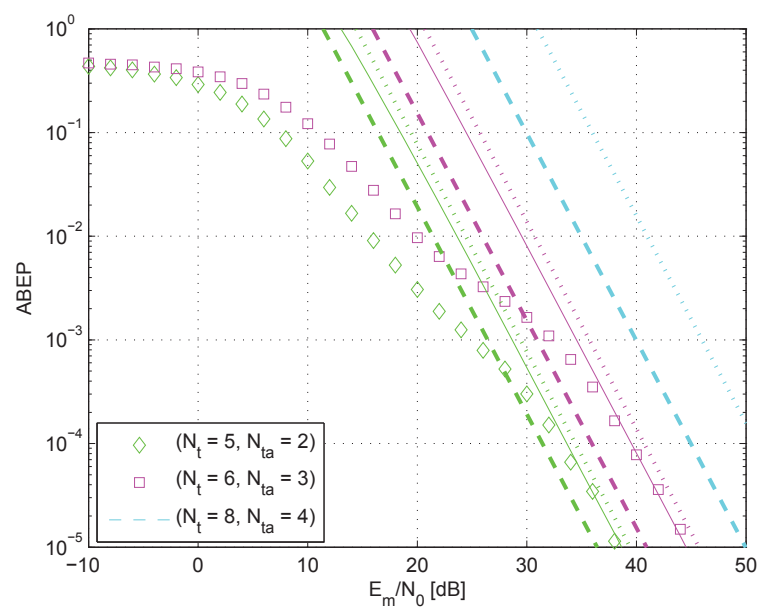

Fig. 13. ABEP of GSSK modulation with multi-user detection. Setup: $N_{u}=$ $3 ; \sigma_{1}^{2}=1$ and $\sigma_{i}^{2}=10^{-2}$ for $i=2,3, \ldots, N_{u} ; N_{r}=2$. Markers show Monte Carlo simulations and solid lines the analytical model (i.e., the formula in the second row of Table II). The ABEP of user 1 (probe/intended link) is shown. Furthermore, dashed lines show the estimated lower-bound (i.e., $\mathrm{ABEP}_{u}^{L L}$ in (23)) and dotted lines show the estimated upper-bound (i.e., $\operatorname{ABEP}_{u}^{U} U$ in (23)), which correspond to the bounds when the probe link is the best link.

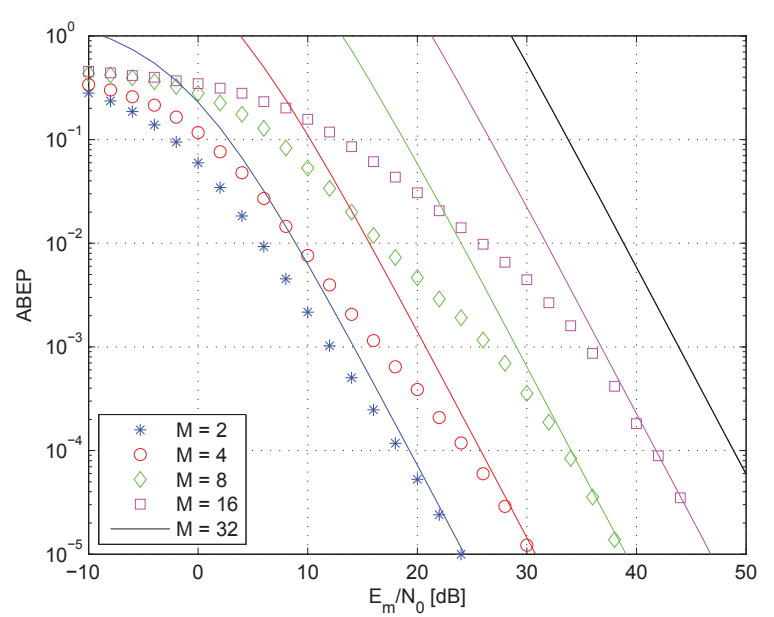

Fig. 14. ABEP of PSK modulation with multi-user detection. Setup: $N_{u}=$ $3 ; \sigma_{1}^{2}=1$ and $\sigma_{i}^{2}=10^{-2}$ for $i=2,3, \ldots, N_{u} ; N_{r}=2$. Markers show Monte Carlo simulations and solid lines the analytical model (i.e., the formula in the first row of Table II). The ABEP of user 1 (probe/intended link) is shown. It is worth mentioning that some simulation results (markers) are not shown due to the long simulation time for medium/high values of $E_{m} / N_{0}$.

about advantages and disadvantages of using the spatialconstellation diagram as a source of information. Clear indications about the system behavior for various channel conditions, interference levels, and MIMO setups have been provided. Comprehensive performance comparisons with conventional modulation schemes for single- and multi-user detection have been given. Overall, our theoretical findings have been well substantiated by Monte Carlo simulations.

Ongoing research is concerned with: i) the extension of the framework to the asynchronous multiple-access scenario and to SM/GSM; ii) the extension of the analysis to frequency- 


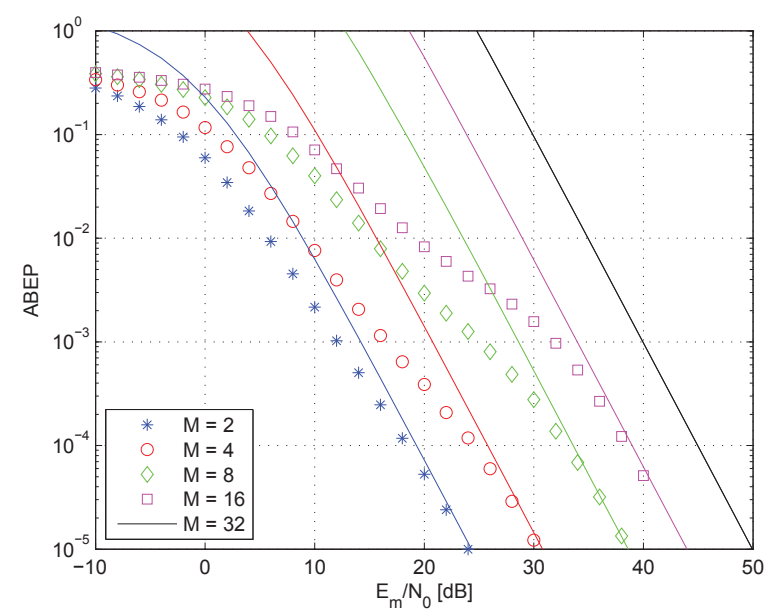

Fig. 15. ABEP of QAM with multi-user detection. Setup: $N_{u}=3 ; \sigma_{1}^{2}=1$ and $\sigma_{i}^{2}=10^{-2}$ for $i=2,3, \ldots, N_{u} ; N_{r}=2$. Markers show Monte Carlo simulations and solid lines the analytical model (i.e., the formula in the first row of Table II). The ABEP of user 1 (probe/intended link) is shown. It is worth mentioning that some simulation results (markers) are not shown due to the long simulation time for medium/high values of $E_{m} / N_{0}$.

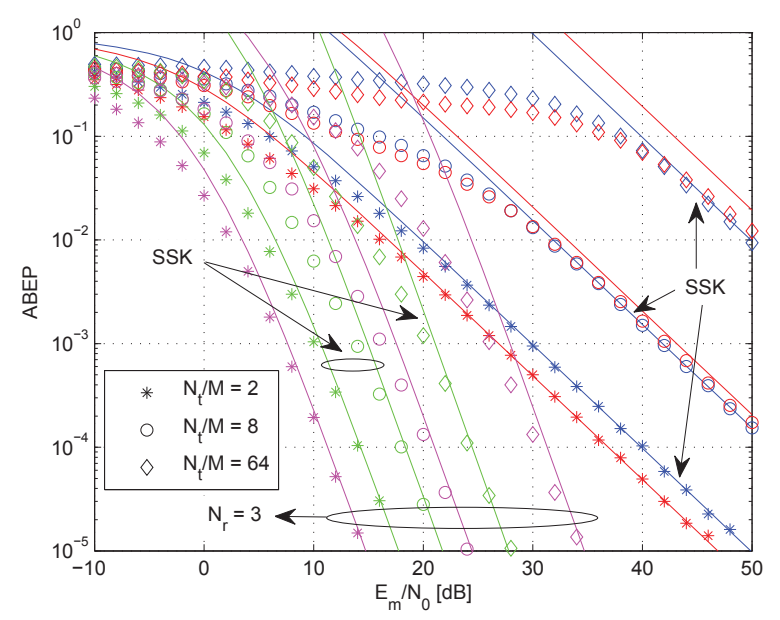

Fig. 16. ABEP of SSK (blue and green lines for $N_{r}=1$ and $N_{r}=3$, respectively) and QAM (red and magenta lines for $N_{r}=1$ and $N_{r}=3$, respectively) modulations with multi-user detection. Setup: $N_{u}=2 ; \sigma_{1}^{2}=1$ and $\sigma_{2}^{2}=5 \times 10^{-2}$. Markers show Monte Carlo simulations and solid lines the analytical model (i.e., (16) and (18) for SSK modulation and the formula in the first row of Table II for QAM). The ABEP of user 1 (probe/intended link) is shown.

selective fading channels, and the possible application of space modulation to Ultra Wide Band (UWB) wireless systems [41], [42], as recently suggested in [2]; iii) the development of simple decoding algorithms, e.g., based on the Sphere Decoding principle [40], for the multi-user detector; and iv) by exploiting the theory of Stochastic Geometry [22], the development and analysis of detectors robust to network interference generated by many randomly distributed interferers.

\section{ACKNOWLEDGMENT}

We gratefully acknowledge support from the European Union (PITN-GA-2010-264759, GREENET project) for this

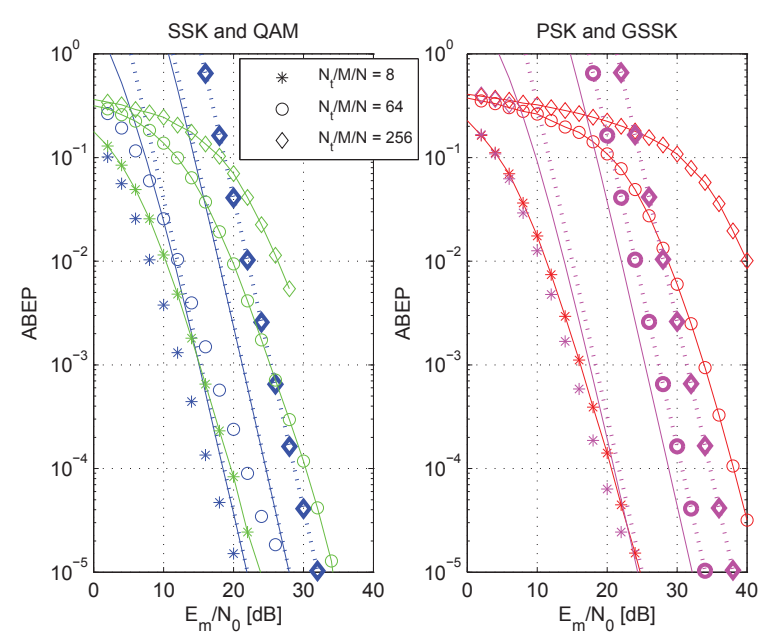

Fig. 17. ABEP of, on the left, SSK (blue curves) and QAM (green curves), and, on the right, PSK (red curves) and GSSK (magenta curves) modulations with multi-user detection. Setup: $N_{u}=2 ; \sigma_{1}^{2}=1$ and $\sigma_{2}^{2}=10^{-2}$; $N_{r}=3$. For GSSK modulation we have: $\left(N_{t}, N_{\mathrm{ta}}\right)=(5,2)$ if $N=8$; $\left(N_{t}, N_{\mathrm{ta}}\right)=(8,4)$ if $N=64$; and $\left(N_{t}, N_{\mathrm{ta}}\right)=(11,4)$ if $N=256$. For SSK and GSSK modulations, markers show Monte Carlo simulations and solid lines the analytical model (i.e., (16) and (18) for SSK modulation and the formula in the second row of Table II for GSSK modulation). For QAM and PSK modulations, only Monte Carlo simulations (markers plus solid lines) are shown for ease of readability. The ABEP of user 1 (probe/intended link) is shown. Also, dashed lines and dashed lines plus markers show the performance estimated through the bounds (i.e., $\operatorname{ABEP}_{u}^{U}$ in (22) for SSK modulation and $\mathrm{ABEP}_{u}^{U U}$ in (23) for GSSK modulation). More specifically: for SSK modulation, dashed lines show the estimated upper-bound if $N=8$ and $N=64$, and dashed lines with markers the upper-bound if $N=8$; and, for GSSK modulation, dashed lines show the estimated upper-bound if $N=8$ and dashed lines with markers the upper-bound if $N=64$ and $N=256$. This is done to improve the readability of the figure. Note that, for GSSK modulation the estimated lower-bound corresponds exactly to the upper-bound computed for SSK modulation and shown on the left-hand side of the figure. It is worth mentioning that some simulation results (markers) are not shown due to the long simulation time for medium/high $E_{m} / N_{0}$.

work. M. Di Renzo acknowledges support of the Laboratory of Signals and Systems (L2S) under the research project "Jeunes Chercheurs". H. Haas acknowledges the EPSRC (EP/G011788/1) and the Scottish Funding Council support of his position within the Edinburgh Research Partnership in Engineering and Mathematics.

\section{REFERENCES}

[1] Y. Chau and S.-H. Yu, "Space modulation on wireless fading channels", IEEE Veh. Technol. Conf., vol. 3, pp. 1668-1671, Oct. 2001.

[2] J. Jeganathan, A. Ghrayeb, L. Szczecinski, and A. Ceron, "Space shift keying modulation for MIMO channels", IEEE Trans. Wireless Commun., vol. 8, no. 7, pp. 3692-3703, July 2009.

[3] M. Di Renzo and H. Haas, "A general framework for performance analysis of space shift keying (SSK) modulation for MISO correlated Nakagami- $m$ fading channels", IEEE Trans. Commun., vol. 58, no. 9, pp. 2590-2603, Sep. 2010.

[4] H. Haas, E. Costa, and E. Schultz, "Increasing spectral efficiency by data multiplexing using antennas arrays", IEEE Int. Symp. Personal, Indoor, Mobile Radio Commun., vol. 2, pp. 610-613, Sep. 2002.

[5] Y. Yang and B. Jiao, "Information-guided channel-hopping for high data rate wireless communication", IEEE Commun. Lett., vol. 12, no. 4, pp. 225-227, Apr. 2008.

[6] R. Y. Mesleh, H. Haas, S. Sinanovic, C. W. Ahn, and S. Yun, "Spatial modulation”, IEEE Trans. Veh. Technol., vol. 57, no. 4, pp. 2228-2241, July 2008 . 
[7] J. Jeganathan, A. Ghrayeb, and L. Szczecinski, "Spatial modulation: Optimal detection and performance analysis", IEEE Commun. Lett., vol. 12, no. 8, pp. 545-547, Aug. 2008.

[8] J. Jeganathan, A. Ghrayeb, and L. Szczecinski, "Generalized space shift keying modulation for MIMO channels", IEEE Int. Symp. Personal, Indoor, Mobile Radio Commun., pp. 1-5, Sep. 2008.

[9] M. M. Ulla Faiz, S. Al-Ghadhban, and A. Zerguine, "Recursive leastsquares adaptive channel estimation for spatial modulation systems", IEEE Malaysia Int. Conf. Commun., pp. 1-4, Dec. 2009.

[10] R. Y. Mesleh, M. Di Renzo, H. Haas, and P. M. Grant, "Trellis coded spatial modulation", IEEE Trans. Wireless Commun., vol. 9, no. 7, pp. 2349-2361, July 2010

[11] M. Di Renzo and H. Haas, "Performance analysis of spatial modulation", IEEE Int. Conf. Commun. Netw. in China, pp. 1-7, Aug. 2010.

[12] S. Sugiura, S. Chen, and L. Hanzo, "Coherent and differential spacetime shift keying: A dispersion matrix approach", IEEE Trans. Commun. vol. 58, no. 11, pp. 3219-3230, Nov. 2010

[13] M. Di Renzo and H. Haas, "Space shift keying (SSK-) MIMO over correlated Rician fading channels: Performance analysis and a new method for transmit-diversity", IEEE Trans. Commun., vol. 59, no. 1 , pp. 116-129, Jan. 2011.

[14] E. Basar, U. Aygolu, E. Panayirci, and H. V. Poor, "Space-time block coded spatial modulation”, IEEE Trans. Commun., vol. 59, no. 3, pp. 823-832, Mar. 2011.

[15] S. Sugiura, S. Chen, and L. Hanzo, "Generalized space-time shift keying designed for flexible diversity-, multiplexing- and complexitytradeoffs", IEEE Trans. Wireless Commun., vol. 10, no. 4, pp. 1144 1153, Apr. 2011.

[16] M. Di Renzo and H. Haas, "Space shift keying (SSK) modulation with partial channel state information: Optimal detector and performance analysis over fading channels", IEEE Trans. Commun., vol. 58, no. 11 , pp. 3196-3210, Nov. 2010

[17] M. Di Renzo and H. Haas, "Bit error probability of space modulation over Nakagami-m fading - Asymptotic analysis", IEEE Commun. Lett., Aug. 2011 (to appear). [Online]. Available: http://ieeexplore.ieee.org/stamp/stamp.jsp?tp=\&arnumber=5992700

[18] V. R. Cadambe and S. A. Jafar, "Interference alignment and the degree of freedom for the $\mathrm{K}$-user interference channel", IEEE Trans. Inform. Theory, vol. 54, no. 8, pp. 3425-2441, Aug. 2008.

[19] F. Khan and J. Pi, "Millimeter-wave mobile broadband: Unleashing 3-300GHz spectrum", IEEE Wireless Commun. Netw. Conf., Mar. 2011, tutorial presentation. [Online]. Available: http://www.ieeewenc.org/2011/tut/t1.pdf.

[20] S. Rajagopal, S. Abu-Surra, Z. Pi, and F. Khan, "Antenna array design for multi-Gbps mmWave mobile broadband communication", IEEE Global Commun. Conf., pp. 1-6, Dec. 2011 (to appear).

[21] S. Verdú, Multiuser Detection, Cambridge University Press, New York, 1998.

[22] M. Z. Win, P. C. Pinto, and L. A. Shepp, "A mathematical theory of network interference and its applications" Proc. of the IEEE, vol. 97 no. 2, pp. 205-230, Feb. 2009

[23] M. P. Lotter and P. Van Rooyen, "An overview of space division multiple access techniques in cellular systems" IEEE South African Symp. Commun. and Signal Proces., pp. 161-164, Sep. 1998.

[24] B. Suard, X. Guanghan, L. Hui, and T. Kailath, "Uplink channel capacity of space-division-multiple-access schemes" IEEE Trans. Inform. The ory, vol. 44, no. 4, pp. 1468-1476, July 1998.

[25] L.-L. Yang and L. Hanzo, "Performance of space-division multipleaccess systems communicating over Nakagami fading channels" IEEE Veh. Technol. Conf. - Spring, pp. 275-279, May 2004

[26] R. L. de Lacerda Neto, A. Menouni Hayar, and M. Debbah, "Channel division multiple access" IEEE Int. Conf. Wireless Broadband and Ultra Wideband Commun., New South Wales, Australia, Mar. 2006.

[27] H. L. Van Trees, Detection, Estimation, and Modulation Theory, Part I, John Wiley \& Sons, Inc. 2001, ISBNs: 0-471-09517-6.

[28] M. Irshid and I. Salous, "Bit error probability for coherent M-ary PSK systems" IEEE Trans. Commun., vol. 39, no. 3, pp. 349-352, Mar. 1991

[29] M. K. Simon and M.-S. Alouini, Digital Communication over Fading Channels: A Unified Approach to Performance Analysis, John Wiley \& Sons, Inc., 1st ed., 2000.

[30] J. Proakis, Digital Communications, McGraw-Hill, 4th ed., 2000.

[31] M. Di Renzo and H. Haas, "Improving the performance of space shift keying (SSK) modulation via opportunistic power allocation", IEEE Commun. Lett., vol. 14, no. 6, pp. 500-502, June 2010.

[32] B. Hassibi and H. Vikalo, "On the sphere decoding algorithm: Part I Expected complexity" IEEE Trans. Signal Process., vol. 53, no. 8, pp. 2806-2818, Apr. 2005.
[33] M. Dohler, Virtual Antenna Arrays, Ph.D. Thesis, King's College London, London, UK, Nov. 2003

[34] T. Weber, I. Maniatis, A. Sklavos, Y. Liu, E. Costa, H. Haas, and E. Schulz, "Joint transmission and detection integrated network (JOINT), a generic proposal for beyond $3 \mathrm{G}$ systems", Int. Conf. Telecommun., pp. 479-483, June 2002.

[35] A. F. Molisch, et al., "Base station cooperation", IEEE 802.16 Broadband Wireless Access WG, IEEE C802.16m-08/817, July 2008

[36] C. Jandura, P. Marsch, A. Zoch, and G. P. Fettweis, "A testbed for cooperative multi cell algorithms", ACM Tridentcom, Mar. 2008.

[37] J. Zhang and J. G. Andrews, "Distributed antenna systems with randomness", IEEE Trans. Wireless Commun., vol. 7, no. 9, pp. 3636-3646, Sep. 2008

[38] V. Jungnickel, et al., "Coordinated multipoint trials in the downlink", IEEE Broadband Wireless Access Workshop, pp. 1-7, Nov. 2009.

[39] J. Mietzner, R. Schober, L. Lampe, W. H. Gerstacker, and P. A Hoeher, "Multiple-antenna techniques for wireless communications A comprehensive literature survey", IEEE Commun. Surveys Tuts., vol. 11, no. 2, pp. 87-105, 2nd quarter 2009.

[40] A. Younis, M. Di Renzo, R. Y. Mesleh, and H. Haas, "Sphere decoding for spatial modulation" IEEE Int. Commun. Conf., pp. 1-6, June 2011

[41] M. Di Renzo, F. Graziosi, and F. Santucci, "A framework for the analysis of UWB receivers in sparse multipath channels with intra-pulse interference via Padé expansion", IEEE Trans. Commun., vol. 56, no. 4 , pp. 535-541, Apr. 2008.

[42] M. Di Renzo, L. A. Annoni, F. Graziosi, and F. Santucci, "A novel class of algorithms for timing acquisition for differential transmitted reference ultra wide band receivers - Architecture, performance analysis and system design", IEEE Trans. Wireless Commun., vol. 7, no. 6, pp. 2368-2387, June 2008

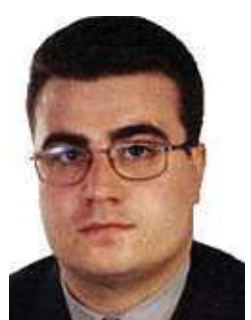

Marco Di Renzo (SM'05-AM'07-M'09) was born in L'Aquila, Italy, in 1978. He received the Laurea (cum laude) and the Ph.D. degrees in Electrical and Information Engineering from the Department of Electrical and Information Engineering, University of L'Aquila, Italy, in April 2003 and January 2007. respectively. From August 2002 to January 2008, he was with the Center of Excellence for Research DEWS, University of L'Aquila, Italy. From February 2008 to April 2009, he was a Research Associate with the Telecommunications Technological Cente of Catalonia (CTTC), Barcelona, Spain. From May 2009 to December 2009 , he was a Research Fellow with the Institute for Digital Communications (IDCOM), The University of Edinburgh, Edinburgh, United Kingdom (UK).

Since January 2010, he has been a Tenured Researcher ("Chargé de Recherche Titulaire") with the French National Center for Scientific Research (CNRS), as well as a research staff member of the Laboratory of Signals and Systems (L2S), a joint research laboratory of the CNRS, the École Supérieure d'Électricité (SUPÉLEC), and the University of Paris-Sud XI, Paris, France. His main research interests are in the area of wireless communications theory, signal processing, and information theory.

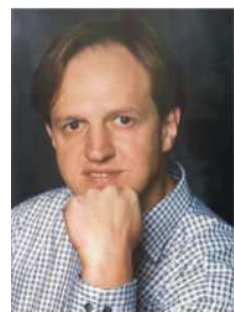

Harald Haas (SM'98-AM'00-M'03) received the Ph.D. degree from the University of Edinburgh in 2001. His main research interests are in the area of wireless system design/analysis and digital signa processing, with a particular focus on interference coordination in wireless networks, spatial modulation and visible light communications. He joined the International University Bremen (Germany), now Jacobs University Bremen, in September 2002 where he is now Honorary Professor of electrical engineering. In June 2007, he joined the University of Edinburgh (Scotland/UK) where he is Professor of Mobile Communications in the Institute for Digital Communications (IDCOM)

Professor Haas has been invited to present his work on visible light communications at the TED conference in 2011. Since 2007, he has been Regular High Level Visiting Scientist supported by the Chinese "111 program" at Beijing University of Posts and Telecommunications. 